Research Paper

\title{
The Implications of Dimensions of E-Government Readiness in Achieving Sustainable Development: A case study in the Nineveh Governorate Bureau
}

Journal of

\section{TANMIYAT AL- RAFIDAIN}

\section{(TANRA)}

A scientific, quarterly, international, open access, and peer-reviewed journal

Vol. 40, No. 131

September 2021

(C) University of Mosul |

College of Administration and

Economics, Mosul, Iraq.

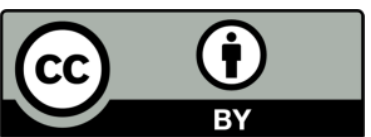

TANRA retains the copyright of published articles, which is released under a "Creative Commons Attribution License for CC-BY-4.0" enabling the unrestricted use, distribution, and reproduction of an article in any medium, provided that the original work is properly cited.

Citation: Al-Aarajy Abdullah Hashim Mohammed, Al-Samman Their Ahmed Saadoon, (2021). "The Implications of Dimensions of E-Government Readiness in Achieving Sustainable Development: A case study in the Nineveh Governorate". TANMIYAT AL-RAFIDAIN, 40 (131), 21 -39, https://doi.org/ 10.33899/tanra.2021.129354.10 69

P-ISSN: 1609-591X e-ISSN: 2664-276X

tanmivat mosulinurnals.com

\section{Abdullah Hashim Mohammed Al-Aarajy 1ः Thaeir Ahmed Saadoon Al-Samman² \\ ${ }^{2 \& 1}$ College of Administration And Economics University of Mosul}

Corresponding author: Abdullah Hashim Mohammed Al-Aarajy, College of Administration And Economics University of Mosul, abdullahalaara86@gmail.com

DOI: https://doi.org/ 10.33899/tanra.2021.129354.1069

Article History: Received: 18/1/2021; Revised: 25/2/2021; Accepted: 4/2/2021; Published: 1/9/2021.

\section{Abstract}

The study aims to show the role of e-government through its dimensions in achieving sustainable development, and the extent of the interest of the researched institution (the Nineveh Governorate Bureau) in these two variables, and to know the level of feeling of employees in the researched institution to the extent of availability of E-Government Readiness Dimensions, and the extent of their agreement on its role in achieving sustainable development, and the Nineveh Governorate Bureau was chosen as a field for research and it included a random sample of the bureau's employees, as the questionnaire form and personal interviews were approved in collecting data, and the questionnaire form included phrases related to the study variables, and (247) electronic forms were collected. Personal interviews were conducted with some leaders and employees in the researched institution who are related to the subject of the study to ensure the comprehensiveness and objectivity of the data collected from the researched institution, and the study relied on the descriptive and analytical approach, and several statistical tools were used, as the study reached a group Among the most important results are: the existence of a correlation and positive impact of the dimensions of E-Government Readiness in achieving Sustainable Development.

Keywords:

E-government Readiness, Sustainable Development. 


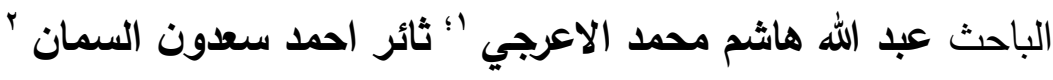
|\& جامعة الموصل، كلية الإدارة والاقتصاد

المؤلف المراسل الباحث عبد الله هاشم محمد الاعرجي ، جامعة الموصل، العراق، abdullahalaara86@gmail.com

DOI: https://doi.org/ 10.33899/tanra.2021.129354.1069

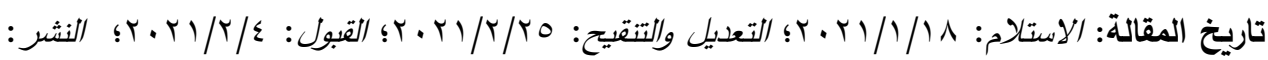
$\cdot r \cdot r / 9 / 1$

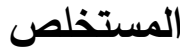

تهاف الدراسة إلى إظهار دور الحكومة الالكترونية من خلال أبعادها في تحقيق التتمية المستدامة،

وبيان مدى اهتمام المؤسسة المبحوثة (ديوان محافظة نينوى) بهذين الهتغيرين، ومعرفة مستوى شعور الموظفين

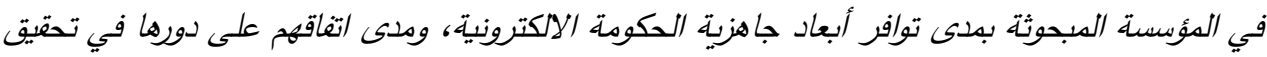

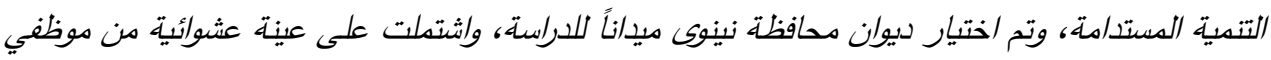

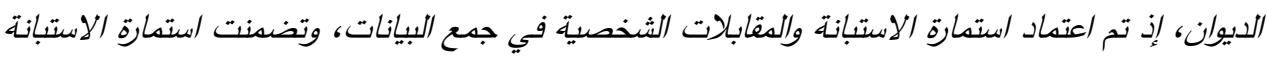

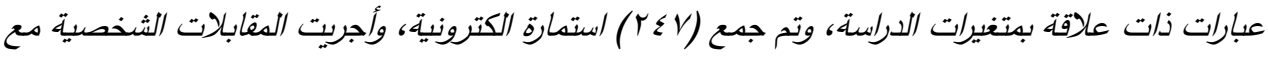
بعض القيادات والموظفين في المؤسسة المبحوثة من ذوي العلاقة بوضوضوع الدراسة للتأكد من شعولية

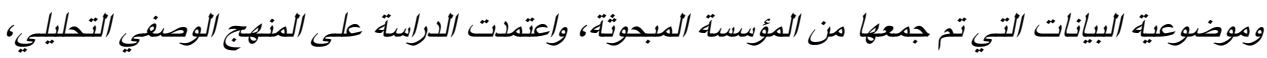

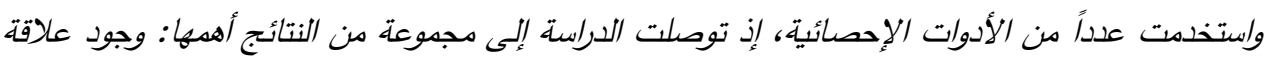

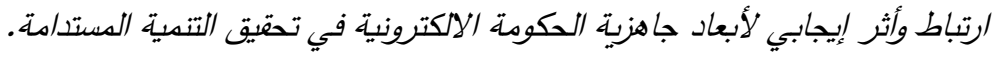

الكلمات الرئيسة

التنظيمية؛ الموارد البثرية؛ التكنولوجية؛ القانونية

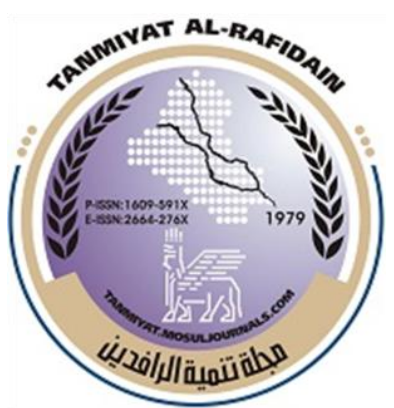

هبلة

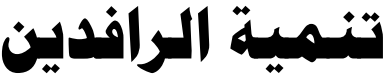

(TANRA) دولية، مفتوحة الوصول، محكمة.

المجلد (• • )، العدد (1T ())،

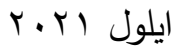

ج جامعة الموصل | كلية الإدارة والاقتصاد، الدوصل، العوصل العراق.

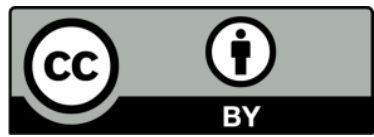

تحتفظ (TANRA) بحقوق الطبع والنشر للمقالات المنشورة، والتي يتم إصدارها بموجب ترخيص لتصن

\lrcorner (Creative Commons Attribution) (CC-BY-4.0) والاستنساخ غير المقيد وتوزيع للمقالة في أي وسيط الأخي نقل، بشرط اقتباس العمل الأصلي بشكل صحيح.

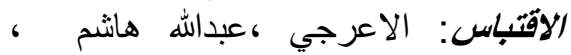

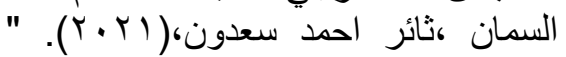

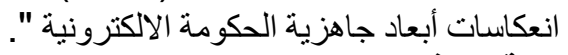

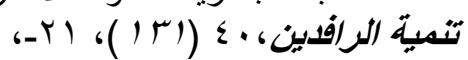
https://doi.org/ 10.33899/tanra.2021.129354.10 69

P-ISSN: 1609-591X e-ISSN: 2664-276X tanmiyat.mosuljournals.com 
يحدد الأنموذج الجديد للحكومات والمتمثل بالحكومة الاككترونية شكل العالم من خلال آثاره التحويلية الكبيرة على القطاعات الاقتصادية والاجتماعية والبيئية وعلى الاتصالات والخدمات، والتي تسهم مباشرة في

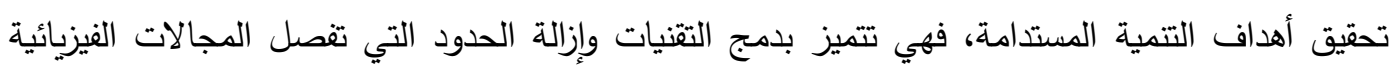
والبيولوجية، إذ تجري حاليا متابعة محاور خطة التنمية المستدامة في سياق التحول القائم على التكنولوجيات

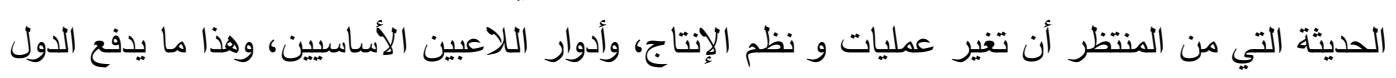

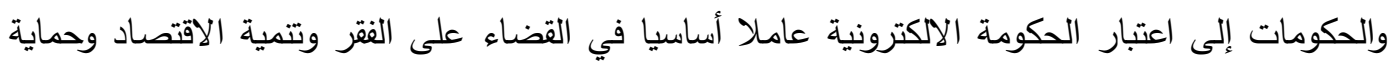
البيئة، فعند استخدام المؤسسات الحكومية لتكنولوجيا المعلومات والاتصالات في التواصل بين الموظفين والإدارات بهدف توفير المعلومات وانتقالها بشكل سريع ومتكامل وذلك وصولاً إلى ترشيد عملية اتخاذ القرار

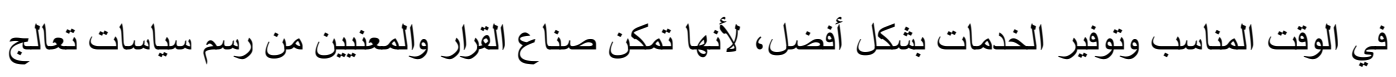
أوجه التفاوت في المجتمع بشكل أفضل ووضع الخطط الاقتصادية الرشيدة، فضلاً عن تثجيع الإجراءات

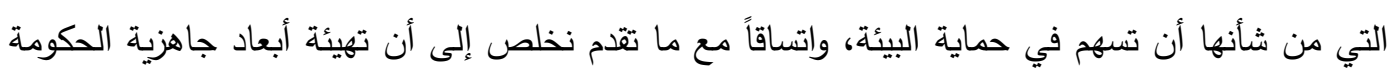

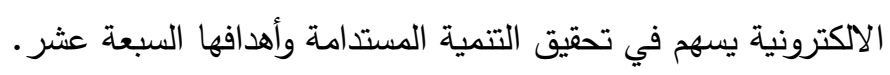

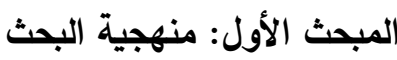

اعتمد الباحثان على المنهج الوصفي التحليلي لغرض اختبار فرضيات الدراسة، وذلك بدراسة العلاقات

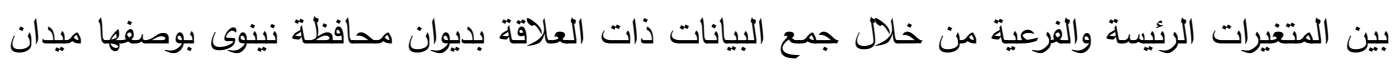

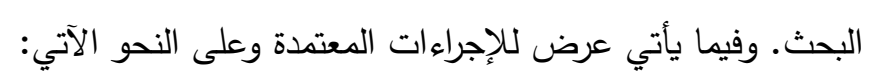
أولاًَ- مشكلة البحث: تهيئة البيئة المناسبة لتنفيذ الحكومة الالكترونية و تثخيص مدى الجئي الجاهزية المتوفرة في المؤسسة (ديوان محافظة نينوى)، وتكثيف جهود الحكومة المركزية والمحلية في ضوء ذلك التوجه من أجل

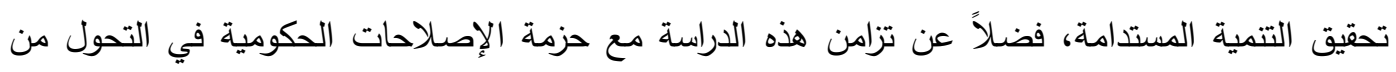
المركزية إلى اللامركزية، وما يتراكم عليها من استحداث مناصب ووحدات إدارية، وإعادة صياغة للهيكل التظيمي بما يتلائم مع الصلاحيات والمسؤوليات التي انتقلت إلى ديوان محافظة نينوى وطبيعة الاتصال البيني مع المديريات التابعة للحكومة المحلية، ولإبراز مشكلة الدراسة بثكل أكثر وضوحاً من خلال طرح الأسئلة الآتية: ا. ـ ما الأبعاد اللازم توافرها لإتمام جاهزية مشروع الحكومة الالكترونية في ديوان محافظة نينوى؟

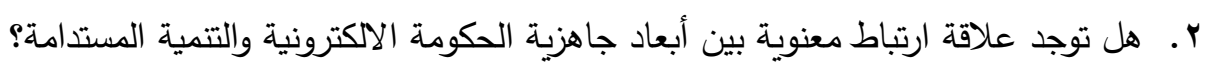

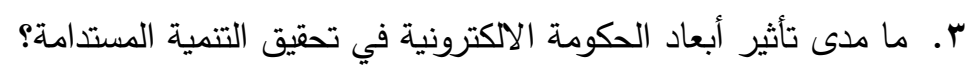

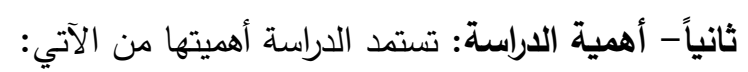

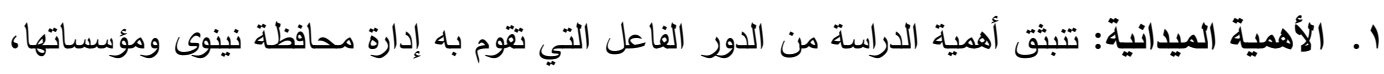
وكذلك في تقديم الخدمات الاجتماعية لمحافظة نينوى، ولكي يكون ديوان محافظة نينوى قادراً على إتمام 
تلك المهام والإجراءات والارتقاء بإدائه تجاه المجتمع، لابد من مواكبة الركب العالمي في التقدم التكنولوجي، ومشاطرة دول العالم في تحقيق أهداف التتمية المستدامة، فلذلك تساهم هذه الدراسة في إبراز لإناء اهمية أبعاد جاهزية الحكومة الاككترونية وضرورة تجهيزها في المؤسسة المبحوثة، والتي تساعد في الهي المضي إلى تحقيق التتمية المستدامة لمحافظة نينوى. r. الأهمية الاكاديمية: تضيف هذه الدراسة معلومات جديدة تعد مرجعاً للباحثين، وفتح آفاق نحو المزيد

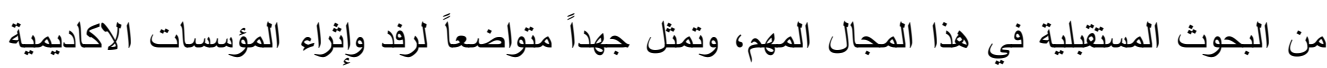
والمكتبات العراقية بثكل خاص والعربية بثكل عام. r. الأهمية الاجتماعية: محاولة تحقيق الوعي وتوسيع مدارك المتمع بأهمية الثقافة الالكترونية، و دور

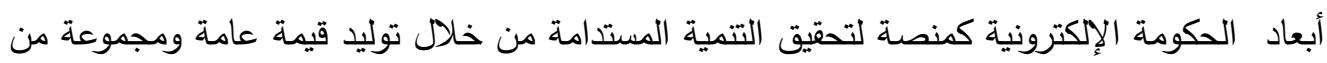
المزايا، والتي تحث المواطنين والمجتمعات المحلية ومنظمات المجتمع المدني على العمل المشترك والمستدام مع المؤسسات الحكومية لتحقيق التتمية المستدامة ومن ثم بناء مجتمع المعرفة. ثالثاً- هدف الدراسة:

إن الغرض من الدراسة الحالية إظهار دور أبعاد جاهزية الحكومة الالكترونية في تحقيق التنمية المستدامة، وبيان مدى اهتمام المؤسسة المبحوثة (ديوان محافظة نينوى) بهذين المتغيرين، والتي تتبثق منها

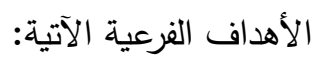
1. بناء إطار معرفي يحيط بموضوعات ( أبعاد جاهزية الحكومة الاككترونية، والتتمية المستدامة) وأبعادهما الفرعية، من خلال عرض الجهود البحثية السابقة في الموضوعات ذات العاديه العلاقة.

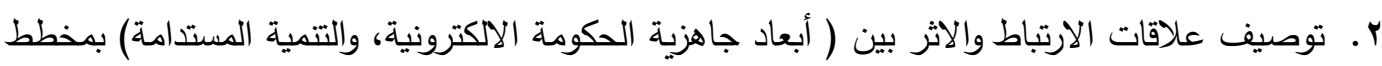
فرضي، واختباره من خلال تحليل طبيعة العلاقات فيما بينها وفق الفرضيات الموضوعة من قبل الباحثين.

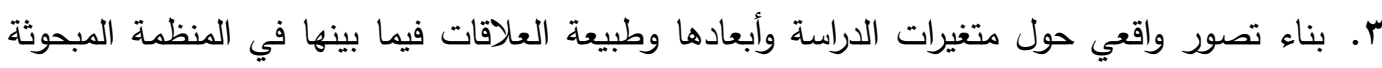
(ديوان محافظة نينوى) من خلال مزاولة أنشطتها وتتفيذ إجراء اتها. رابعاً - فرضيات الدراسة والمخطط الفرضي لها:

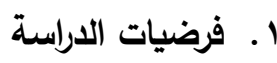
انسجاماً مع مشكلة الدراسة واتساقاً مع أهدافها، تم تحديد مجموعة من الفروض الرئيسة والفرعية للدراسة وكما يأتي:

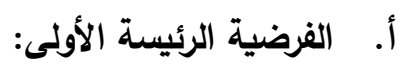
H0.1

$$
\text { مستوى معنوية (م)0.05). }
$$

ومن هذه الفرضية ندرج الفرضيات الفرعية الفرآلاتية: 
الاعرجي والسمان

• H0.1.1: لا توجد علاقة ارتباط بين بعد الجاهزية التظيمية والتتمية المستدامة عند مستوى معنوية

. $(\alpha \leq 0.05)$

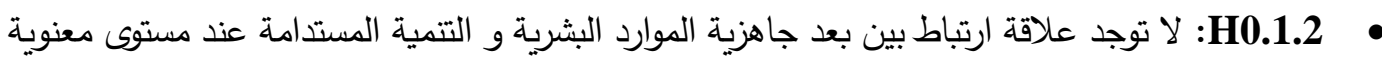

.$(\alpha \leq 0.05)$

• H0.1.3: لا توجد علاقة ارتباط بين بعد الجاهزية التكنولوجية و التتمية المستدامة عند مستوى معنوية

.$(\alpha \leq 0.05)$

• H0.1.4: لا توجد علاقة ارتباط بين بعد الجاهزية القانونية و التمية المستدامة عند مستوى معنوية

. $(\alpha \leq 0.05)$

ب. الفرضية الرئيسة الثانية:

H0.2: لا يوجد أثر لأبعاد جاهزية الحكومة الاككترونية مجتمعة في التتمية المستدامة عند مستوى

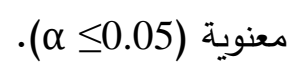

ومن هذه الفرضية ندرج الفرضيات الفرعية الآتية:

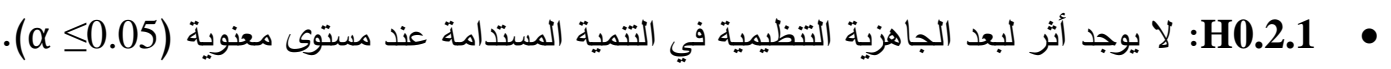

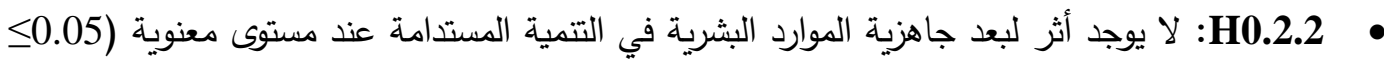

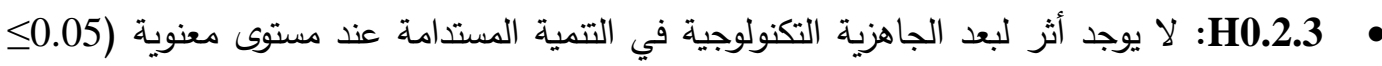

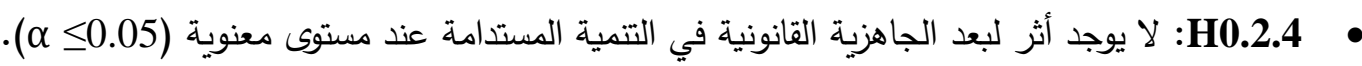

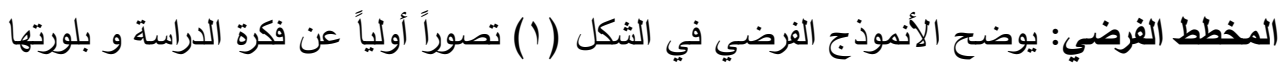
في إطار علاقات الارتباط والأثر المباشرة بين المتغيرات المعتمدة (أبعاد الحكومة الالكترونية، والتتمية المستدامة) وأبعادها الفرعية، والمتمثلة بالآتي: 


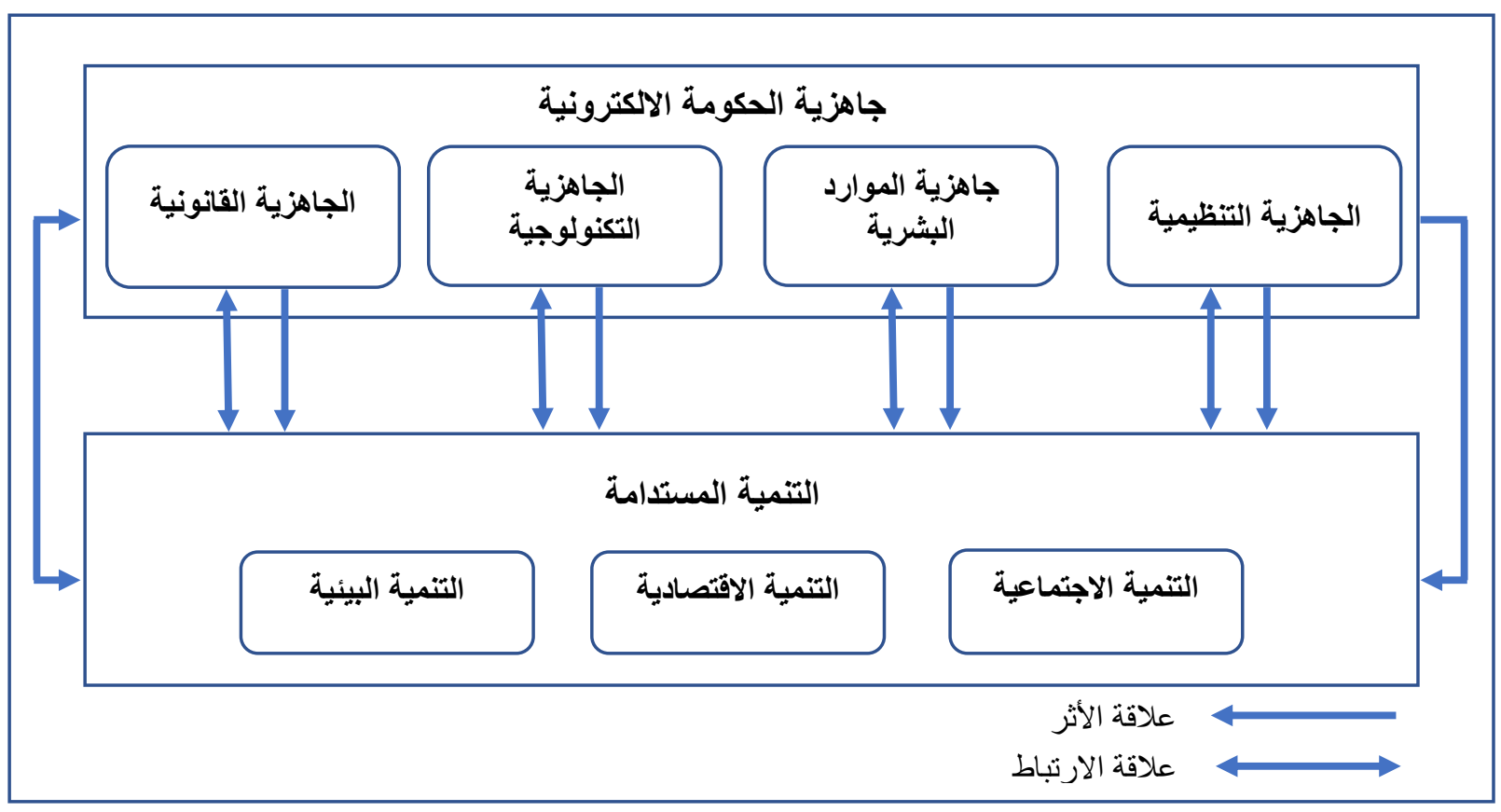

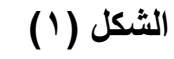

المخطط الفرضي

المصدر: الثكل من إعداد الباحثين

خامساً- حدود الدراسة :تمثلت حدود الاراسة بالآتي:

ا. الحدود المكانية: تم اختيار (ديوان محافظة نينوى) جانباً ميداناً للدراسة الحالية.

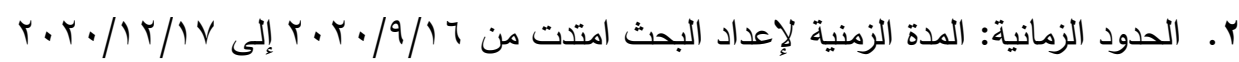

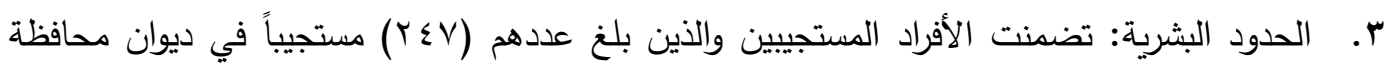

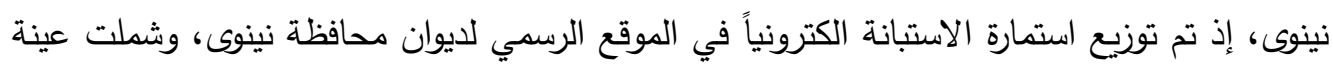
الدراسة (ب97) موظفاً تقريباً متمثلين بالقيادات الإدارية والمستثارين ومديري الأقسام والوحدات الإدارية والموظفين على اختلاف اختصاصاتهم والبالغ. سادساً- أساليب جمع البيانات والمعلومات وتحليلها: بغية الحصول على البيانات اللازمة لمعرفة وتحليل أبعاد الدراسة ومتضمناتها، التي تسهم في اختبار فرضيات الدراسة ومخططها والوصول إلى استنتاجاتها وأهدافها، أعتمد الباحثان على الأدوات البحثية الآتية: أ- المقابلات الشخصية: قام الباحثان بإجراء مقابلات الثخصية مع عدد من المسؤولين والقيادات الإدارية

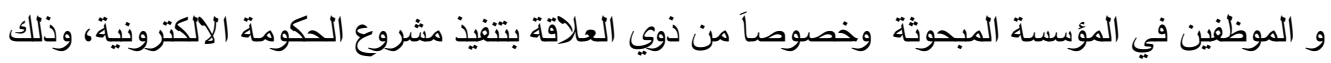
من أجل اقتباس معلومات تقوي من ركائز الدراسة. 


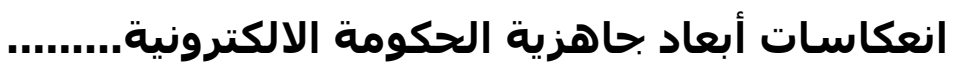

الاعربحي والسمان

ب- استمارة الاستبانة: تعد استمارة الاستبانة الأداة الرئيسة في إطار جمع بيانات الجانب العملي من الدراسة،

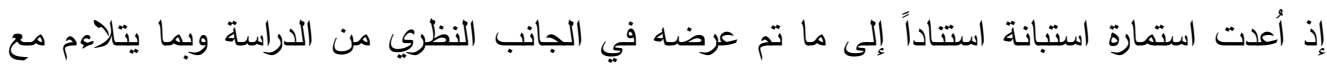
المتغيرات المراد قياسها و ميدان البحث، واعتمد الباحثان على مقياس ليكرت ذي الأوزان الثلاثة والمرتبة

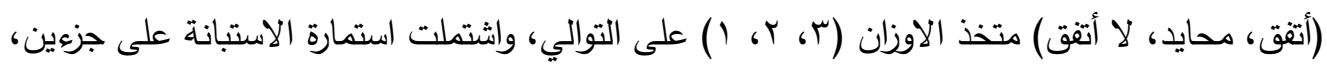

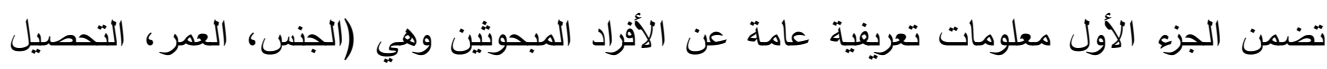
الدراسي، العنوان الوظيفي، سنوات الخدمة)، وتضمن الجزء الثاني مقاييس البحث والتي تم تصنيفها على التى لتصني

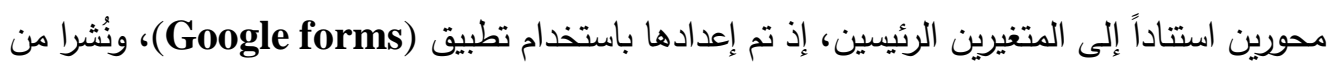

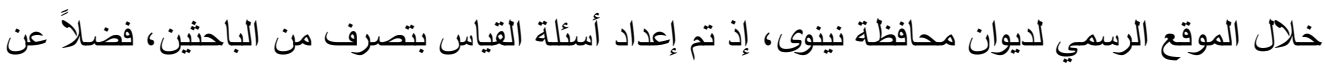

$$
\text { بعض المصادر و الجدول (1) يوضح لرسئ ذلكان }
$$

الجدول (1) رموز متغيرات الدراسة وأبعادها الفرعية في استمارة الاستبانة

\begin{tabular}{|c|c|c|c|c|}
\hline المصادر المعتمدة & مؤشرات القياس & الأبعاد الفرعية & الترميز & المتغير الرئيس \\
\hline \multirow{4}{*}{$\begin{array}{r}\text { (ALbla, } 2018 \\
\text { (AlEisaei, 2019) }\end{array}$} & RO1-RO4 & الجاهزية التتظيمية & \multirow{4}{*}{$\mathrm{RE}$} & \multirow{4}{*}{ لالككومة أبعاد $\quad$ جاهزية } \\
\hline & RH1-RH5 & جاهزيـة الموارد البشرية & & \\
\hline & RT1-RT5 & الجاهزيـة التكنولوجية & & \\
\hline & RL1-RL4 & الجاهزية القانونية & & \\
\hline \multirow{3}{*}{$\begin{array}{l}\text { (Maiye and } \\
\text { McGrath, 2010) } \\
\text { (Hashim and } \\
\text { Abdulhaq, 2019) }\end{array}$} & \multirow{3}{*}{$\begin{array}{l}\text { SD1- } \\
\quad \text { SD5 }\end{array}$} & التتمية البيئية & \multirow{3}{*}{ SD } & \multirow{3}{*}{ المستدامة التنمية } \\
\hline & & التتمية الاجتماعية & & \\
\hline & & التمية الاقتصادية & & \\
\hline
\end{tabular}

المصدر: من إعداد الباحثين

ج- اختبارات بعد التوزيع: بغية التعرف إلى مدى ثبات العبارات لمتغيرات استمارة الاستبانة للظاهرة

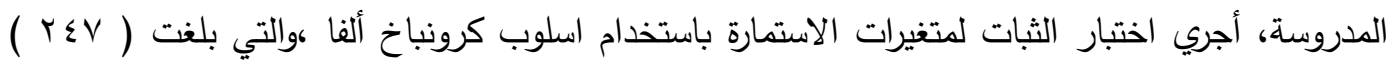

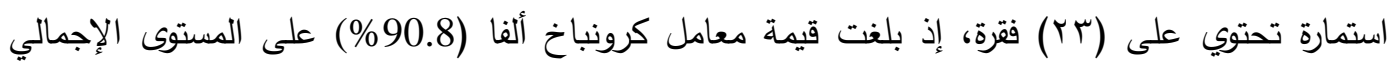
للمتغيرات، وتعد هذه القيمة مقبولة لأغراض البحث، وقد تحققت جودة الأداء، حين زاد معامل كرونباخ ألفا عن الحد الأدنى المقبول وهو (•r\%) (Al-Samman,2008:16)، وهي قيمة مرتفعة وموجبة الإشارة، وهذا يدل على ثبات الاستبانة، وأن جميع فقرات استمارة الاستبانة مهمة وضرورية، وكانت قيم معامل كرونباخ ألفا قد انحصرت ما بين (90.36\%) و (90.86\%) وهي قيم عالية بالنسبة للقيم المقبولة في الدراسات

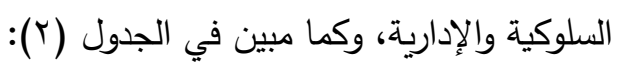

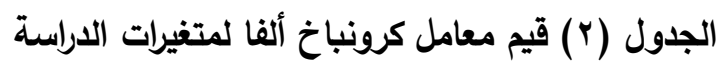




\section{انعكاسات أبعاد بحاهزية الحكومة الالكترونية........................ \\ الاعربحي والسمان}

\begin{tabular}{|c|c|c|c|}
\hline قيمة (Cronbach Alpha) & رموز العبارات & الأبعاد الفرعية & المتغير الرئيسية \\
\hline 0.9080 & RO1-RO4 & الجاهزية التتظيمية & \\
\hline 0.9086 & RH1-RH5 & جاهزية الموارد البشرية & أبعادجاهزية \\
\hline 0.9052 & RT1-RT5 & الجاهزية التكنولوجية & الحكومة الاككترونية \\
\hline 0.9055 & RL1-RL4 & الجاهزيـة القانونية & \\
\hline \multirow{3}{*}{0.9036} & \multirow{3}{*}{ SD1-SD5 } & التتمية الاقتصادية & \multirow{3}{*}{ التتمية المستدامة } \\
\hline & & التمية البيئية & \\
\hline & & التتمية الاجتماعية & \\
\hline
\end{tabular}

المصدر : من إعداد الباحثين.

دـ أساليب التحليل الإحصائي: اعتمد الباحثان على مجموعة من الوسائل وأدوات التحليل الإحصائي وبما

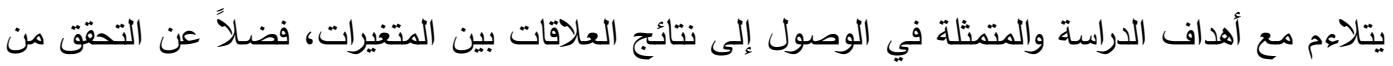

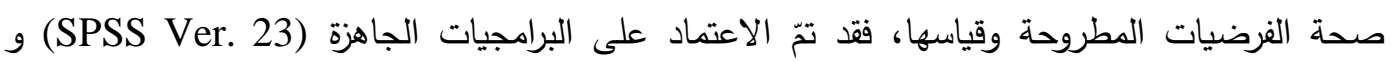
(Amos. v 24)

يأتي:

أ- التكرارات: الغرض منها استعراض إجابات المبحوثين على فقرات استمارة الاستبانة.

ب- النسب المئوية: الغرض منها معرفة نسبة الإجابة لمتغير ما من مجموع الإجابات.

ت- الوسط الحسابي: من خلاله يتم عرض متوسط إجابات المبحوثين لمتغير ما.

ث- الانحراف المعياري: يوضح هذا المقياس درجة تثشت إجابات المبحوثين عن وسطها الحسابي.

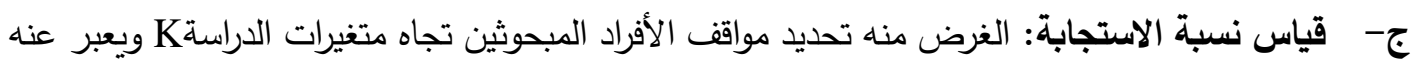

$$
\text { وفق الصيغة الآتية: نسبة الاستجابة= }
$$

هـ - الأدوات الإحصائية المستخدمة لاختبار فرضيات الاراسة، وكما هو موضح أدناه:

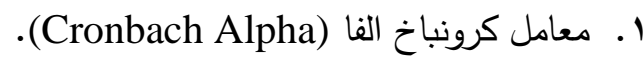

. SPSS V.2 . .

r. نمذجة المعادلات البنائية باستخدام البرنامجين: (SPSS V.23) (AMOS V.24).

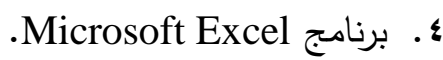

المبحث الثاني: الجانب النظري

E-Government Readiness

أولاًا أبعاد جاهزية الحكومة الاكترونية

إن الاستخدام الفاعل لتكنولوجيا المعلومات والاتصالات يتطلب أن تكون المؤسسة جاهزة الكوانه الكترونياً

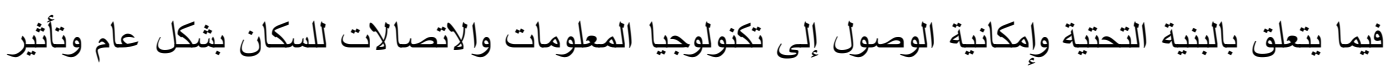

TANMIYAT AL-RAFIDAIN (P-ISSN: 1609-591X; E-ISSN: 2664-276X) تنمية الرافدين 


\section{| \\ الاعربحي والسمان}

الإطار التظيمي لاستخدام تكنولوجيا المعلومات والاتصالات، وتهيئة العاملين للتعامل مع تكنولوجيا

المعلومات والاتصالات، وتوفير المظلة القانونية والتشريعية للإجراءات والأعمال الإكترونية.

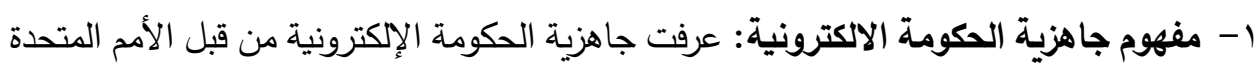

على أنها أهلية الحكومة لاستخدام تكنولوجيا المعلومات والاتصالات لنقل خدماتها وأنثطتها إلى البيئة الجديدة (United Nations,2018:19)، فجاهزية الحكومة الإلكترونية هي مقياس

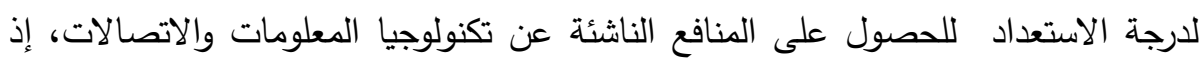
يُستخدم هذا الإجراء غالبًا لقياس مدى استعداد الحكومة للمشاركة في الأنشطة الإلكترونية مثل التجارة الإكترونية والحكومة الإكترونية (1: Dada,2006).

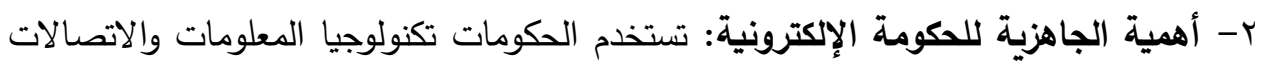
للتواصل بين الحكومات ولتتفيذ الخدمات لإعلام المواطنين والتواصل معهم ، وكذلك لتعزيز

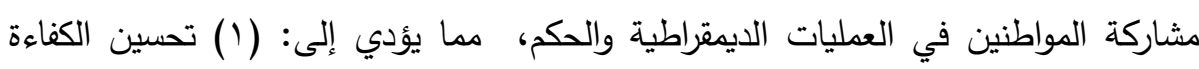
والوصول المريح والأسرع إلى الخدمات الحكومية؛ (Y) زيادة الثفافية ومساءلة موظفي ولئي الحكومة؛ (ץ) انخفاض تكاليف الخدمات الإدارية؛ (ع) تحسين الديمقراطية، إذ تعد تكنولوجيا المعلومات والاتصالات عندئذ أدوات حاسمة في مكافحة الفقر ورفع المستويات الاجتماعية

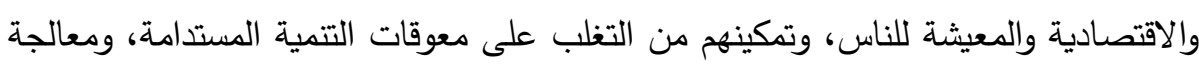

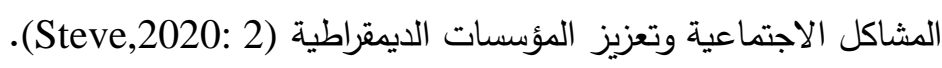
ץ- أهمية تطوير سياسات واستراتيجيات فاعلة للحكومة الإكترونية، ويتطلب ذلك تصميم إطار الإنية

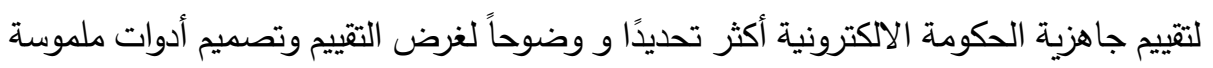

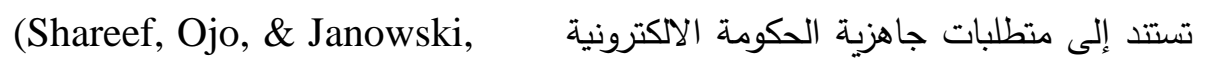

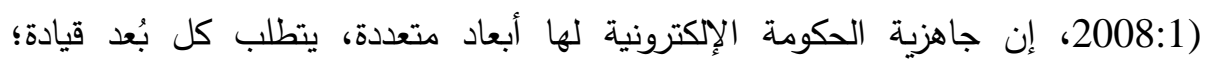
واستراتيجية؛ وتتسيقًا متقاطعًا؛ ومعرفة فنية، وكل ذلك مع استراتيجية تكنولوجية لأخذ الرؤية إلى الواقع (Al-Enzee, 2013:42)، وهذا يوضح أهمية هذه الدراسة في المساهمة في تقييم

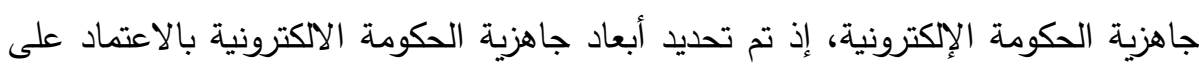

$$
\text { الادبيات السابقة وعلى النحو الآتي: }
$$

أ- الجاهزية التنظيمية: تمثل الجاهزية التظظيمية العمود الفقري للحكومة الالكترونية، وتتمثل بهندرة الهيكل التنظيمي والعمليات الداخلية للمؤسسة بما يتلاءم مع أنثطة الحكومة الالكترونية،

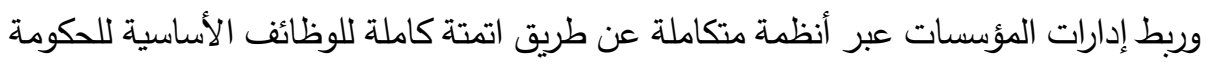
التقليدية، وتثتمل على أنظمة إدارة قواعد البيانات، ونظام مختص بإدارة الموارد البشرية ونظام

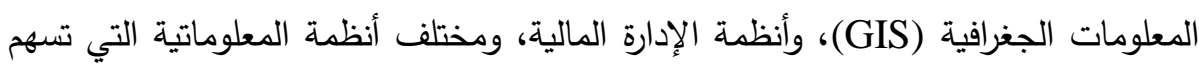
في دعم العمليات الإدارية للحكومة (Bou Marwan, 2014:46). 
ب- جاهزية الموارد البثرية: وتثير إلى وجود موظفين مؤهلين في القطاع العام قادرين على

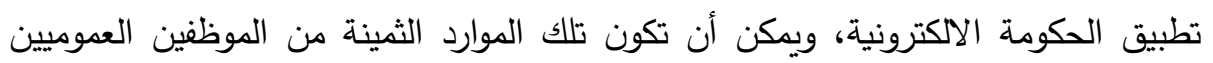

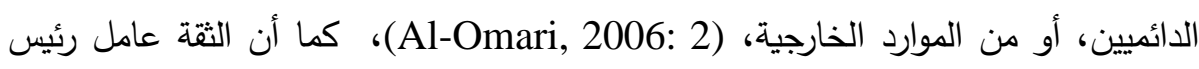
لنجاح المشروعات الحكومة الاككترونية والتي يجب أن تسود داخل المؤسسات الحكومة بأسرها

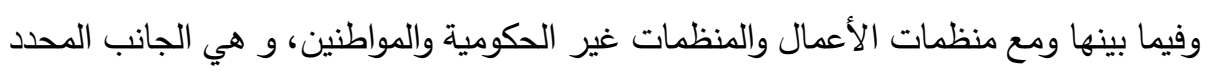

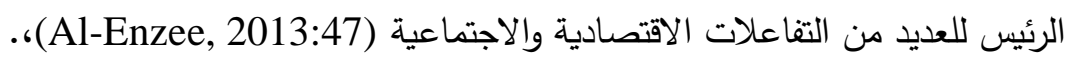

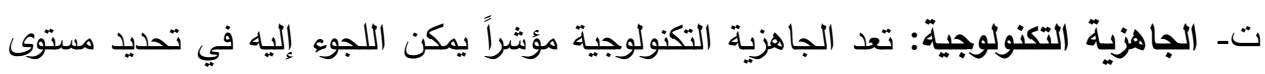

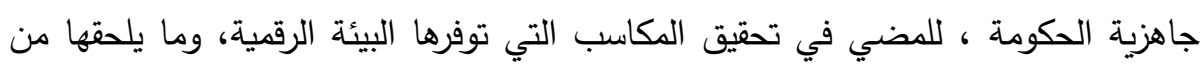

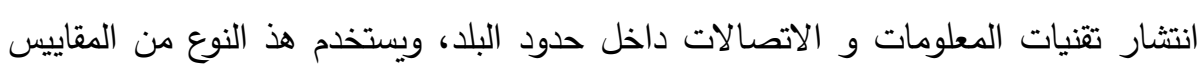
لتحديد مدى استعداد الحكومة للمشاركة في الأنشطة التكنولوجية، والتي تشكل الشريان الحيوي لتصني الذي يرتكز عليه مجتمع المعلومات والمعرفة (Al-Rizo,2012:189) ، كما تثمل الجاهزية التكنولوجية التأكد من تمكين المواطنين للوصول إلى المحتوى الاككتروني، وتوفير كافة أنواع

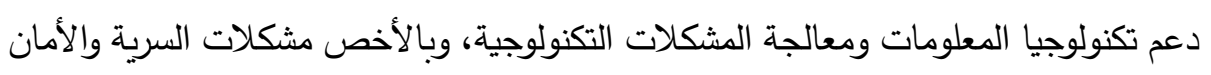

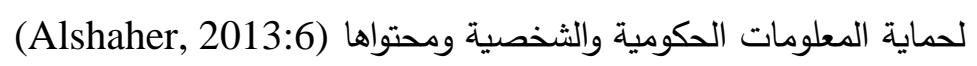
ثـ الجاهزية القانونية: تتجاوز جاهزية الحكومة الإكترونية القضايا التنظيمية و الموارد البشرية

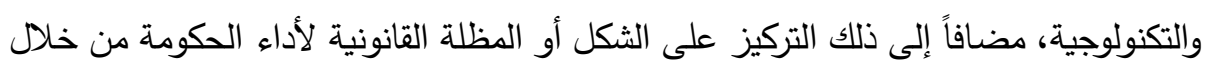

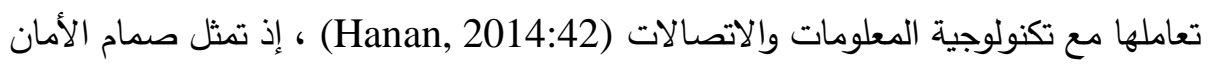
لجميع الأنشطة الحكومية. ويجب على كل مؤسسة ترغب في تتفيذ المبادرة الإلكترونية إجراء تثييم قانوني منفصل لقضيتها، تشمل المسائل القانونية أجزاء كثيرة، أهمها: مشروعية إجراء

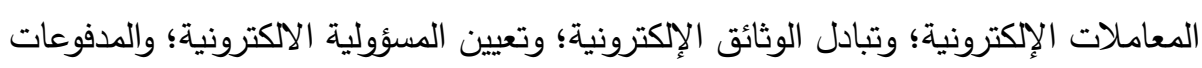

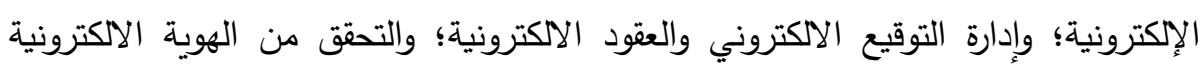

\section{وإجراءات المصادقة الالكترونية (ALbla, 2018:7).}

ثانياً - التنمية المستدامة: التتمية المستدامة في الدول المتقدمة تركز على التخفيض في مستويات استهلاك

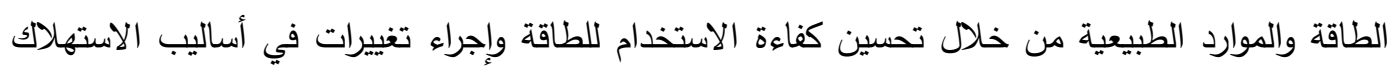
للموارد الطبيعية، ويقع على عاتق البلدان المتقدمة مسؤولية استثنائية في قيادة التتمية المستدامة، لأن تراكمية استهاكها للموارد الطبيعية في الماضي أدت إلى مشكلات كبيرة في التلوث العالمي، إضافة إلى المقدرة

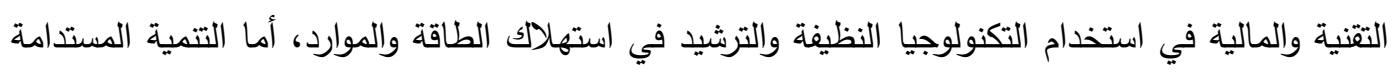
في الدول النامية فتركز على استخدام الموارد بهدف تحسين مستويات المعيشة والتخفيف من الفقر الذي له له ارتباط وثيق بتدهور البيئة وتسارع النمو السكاني (Al-Habal, 2003:4) 
1- مفهوم التنمية المستدامة: تم استخدام مصطلح "التتمية المستدامة" الذي صاغه أول مرة رئيس

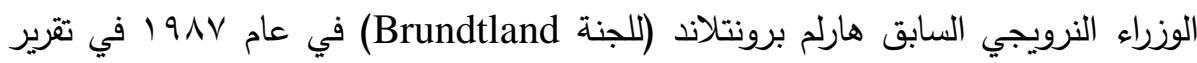
اللجنة العالمية للبيئة والتتمية، بأنها "التتمية التي تلبي احتياجات الحاضر داضدر دون المساس بقدرة

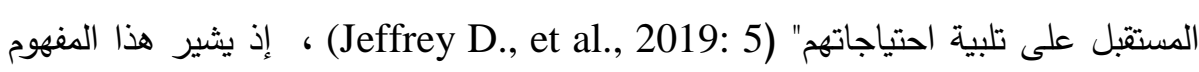
إلى إدارة وصياغة البنى التحتية للموارد الطبيعية والاسترشاد بالتطورات التكنولوجية والمؤسساتية

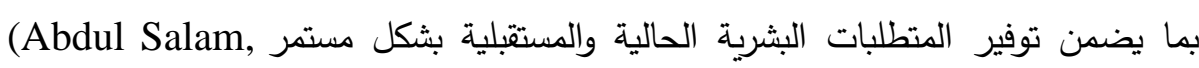
1998:157، بينما يشير البعض الآخر إلى كونها نظرية تقييمية منسقة ومنظمة ومنهجية للسياسة الاقتصادية والعامة، والجهود المبذولة لقياس الاستدامة عن طريق التقدم البشري البري

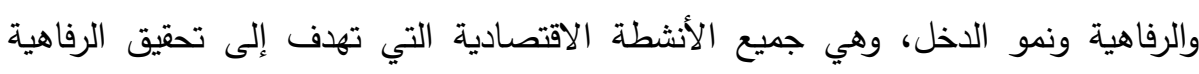

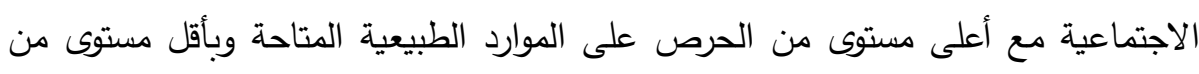

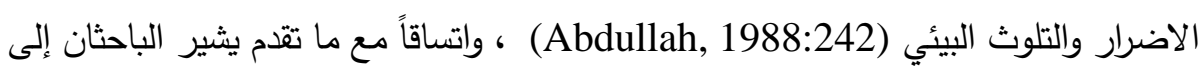

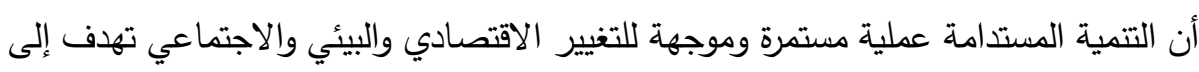
تعزيز رفاهية المواطنين الآن وفي المستقبل، و يتطلب تحقيق التتمية من هذا النوع إنثاء اقتصاد مستدام وفعال من حيث الموارد قائم على مجتمع عادل ومنصف، وفئي يحترم الحدود البيئية

$$
\text { والقدرة على تحمل البيئة الطبيعية. }
$$

r- مبادئ التنمية المستدامة: يجب أن تأخذ جميع برامج التنمية المستدامة في الاعتبار المجالات الثلاثة للاستدامة - البيئة والمجتمع والاقتصاد، إذ تثمل المبادئ التي تقوم عليها الاستدامة

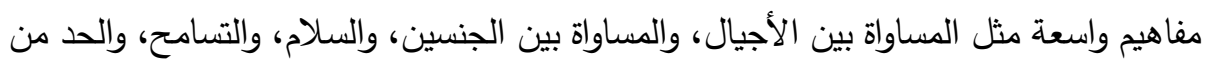

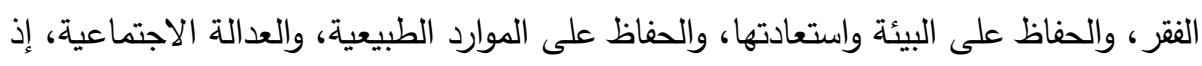

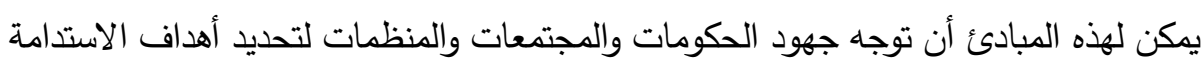
وإنشاء برامج للمساعدة في تحقيق تلك الأهداف، من المهج أن يدرك المعلمون والقادة والمواطنون

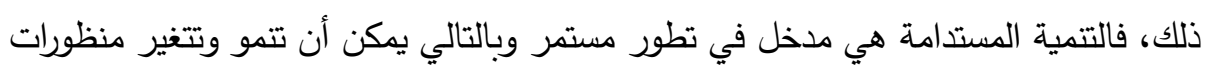
الاستدامة (UNESCO, 2012:

ץ- مجالات التنمية المستدامة: التنمية المستدامة هي مفهوم معياري يجمع بين عدد معين من

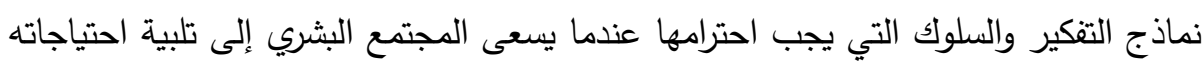

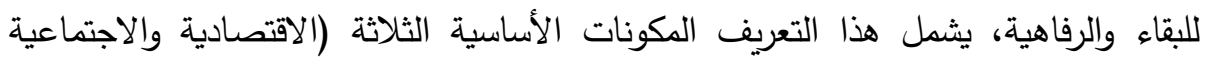
والبيئية) التي تثكل أساس التتمية المستدامة. هذه العناصر الثلاثة مترابطة، لدرجة أن الجهود المبذولة لتعزيز التمية يجب أن تدعم هذا التكوين الثلاثي (Reed, 2003:4)، وفيما يأتي الجوانب الثلاثة للتمية المستدامة: 


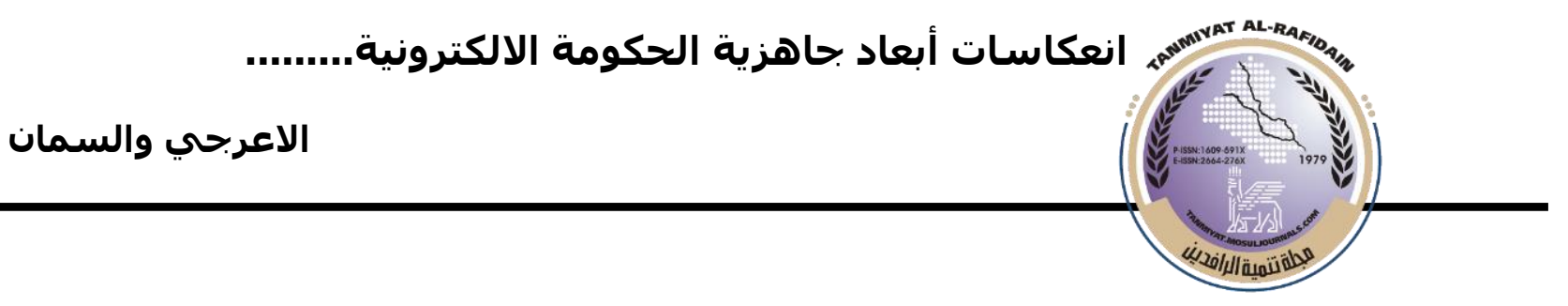

أ- التنمية البيئية: يقوم البعد البيئي للتمية المستدامة على الحاجة إلى الحفاظ على سلامة النظم البيئية

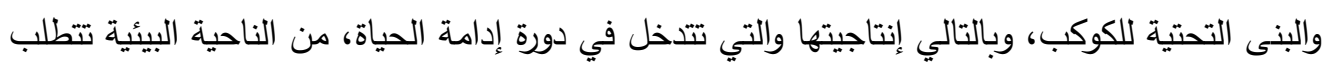

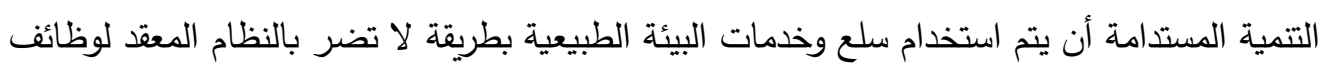

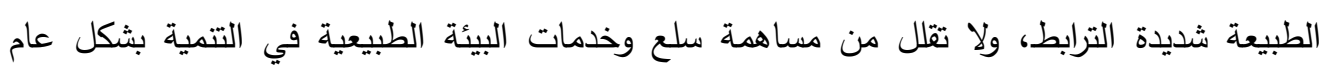

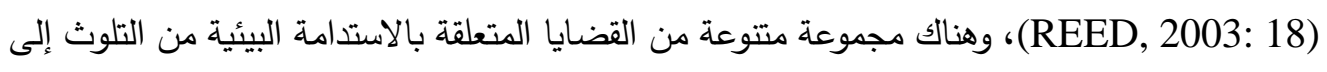

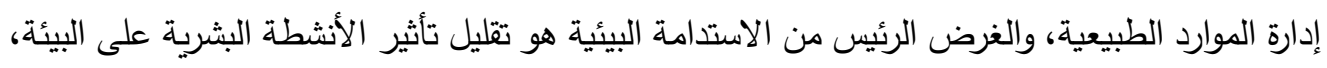

فضلاً عن تثجيع استعادة بيئتا الطبيعية والحفاظ عليها (Maria, L., et. al, 2015: 35). ب- التنمية الاجتماعية: يركز البعد الاجتماعي على الانسان الذي يمثل جوهر التتمية المستدامة من خلاد

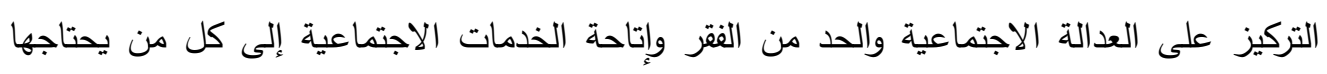

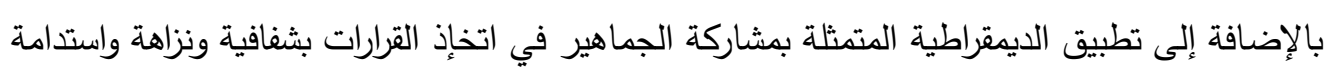
المؤسسات في ظل التتوع الثقافي (Haffaf and Boutiaf, 2015)، وينظر إلى فئل التتمية الاجتماعية

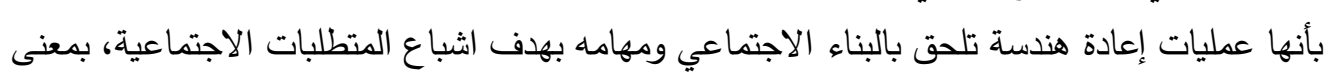

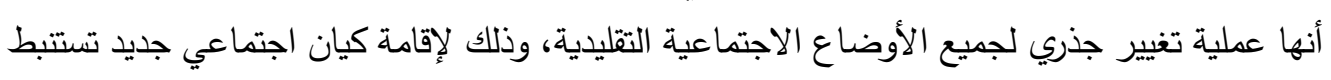

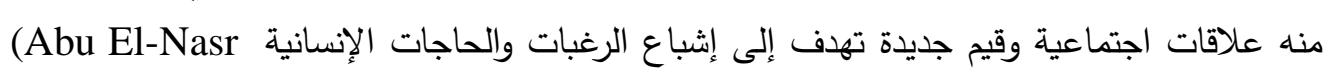
(and Mohamed, 2017:91) فإننا نفهم الاستدامة الاجتماعية كوسيلة لخلق مجتمعات قوية وحيوية وصحية تعزز نوعية الحياة والمرونة العامة، إذ يوفر إنثاء بيئة مبنية عالية الجودة خدمات محلية يمكن الإنية

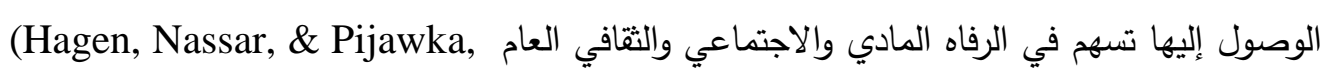
.2017:4)

ت- التنمية الاقتصادية: ويشير البعد الاقتصادي للتتمية المستدامة إلى الإجراءات المنسقة والمستدامة التي

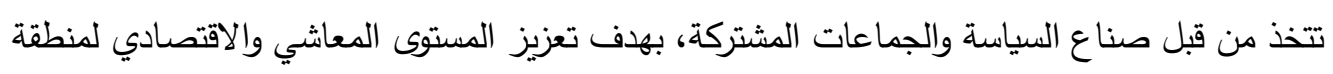

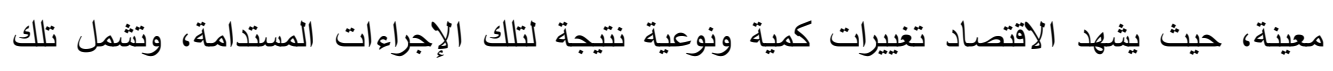

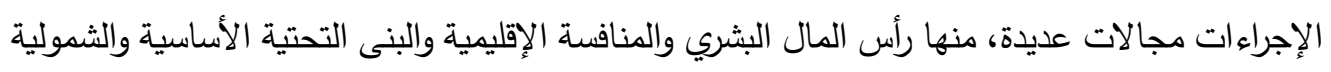
الاجتماعية والامن والصحة والاستدامة البيئية، وتركز التتمية المستدامة على المساعي للتندل في ولئي

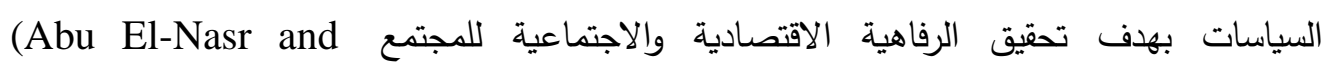

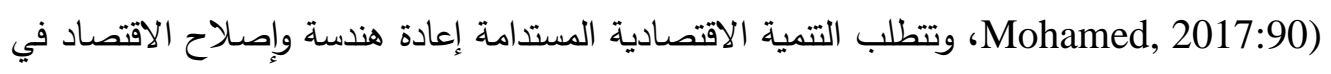

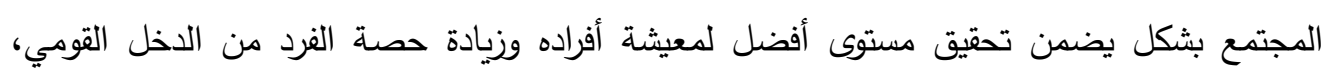
ويتضمن هذا البعد الحد من استتزاف الموارد الطبيعية بإجراء تخفيضات مستمرة في مستويات استهلاك الطاقة من خلال إحداث تغيير جذري وتحسين مستوى الكفاءة في أسلوب الحياة، والحد من أساليب الاستهلاك التي تهدد التتوع البيولوجي (Abu El-Maati, 2014:13 ). المبحث الثالث: الجانب العملي 
المحور الأول: وصف متغيرات الدراسة

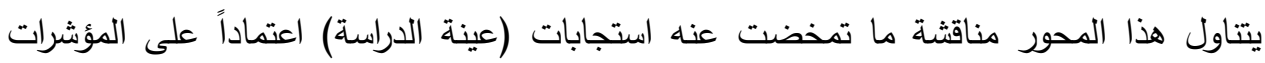
الإحصائية المتمثلة بالنسب المئوية والتكرارات والمتوسطات الحسابية والانحرافات المعيارية المتعلقة بمتغيرات الدراسة وأبعادها الرئيسة وكما يأتي:

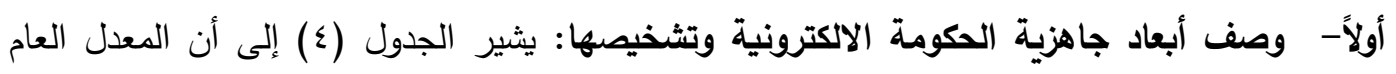

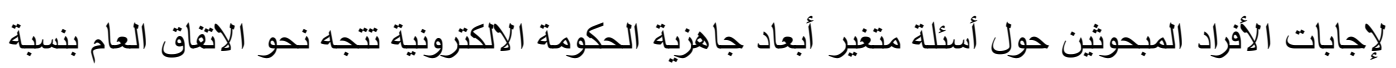

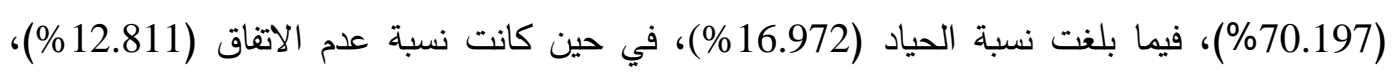
وتعزيزاً لتلك النسب، بلغ الوسط الحسابي العام لهذا المتغير (2.5737) وهو أعلى من الوسط الحسابي

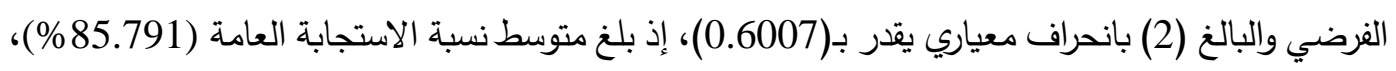

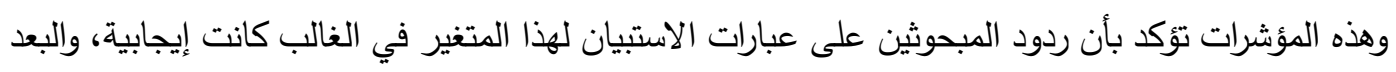

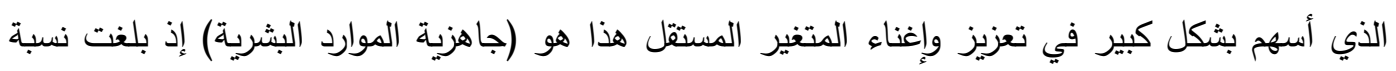
الاتفاق (80.24\%)، وكانت نسبة الاستجابة (90.985\%) وبوسط حسابي يقدر بـ(2.7287) وبانحراف معياري مقدار(0.4972)، إذ تشير إلى اتفاق المبحوثين بدرجة واضحة حول أهمية هذا البعد في تتفيذ الحكومة الالكترونية وضرورة الاهتمام بتطويره.

جدول (ء) المعدل العام والتوزيعات التكرارية والاوساط الحسابية والانحرافات المعيارية ونسبة الاستجابة وضية للمتغير المستقل (أبعاد جاهزية الحكومة الاككترونية)

\begin{tabular}{|c|c|c|c|c|c|c|c|}
\hline \multirow[t]{2}{*}{ نسبة الاستجابة } & \multirow[t]{2}{*}{ الانحراف المعياري } & \multirow[t]{2}{*}{ الوسط الحسابي } & \multicolumn{3}{|c|}{ مقياس الاستجابة } & \multirow[t]{2}{*}{ بعد الترميز } & \multirow[t]{2}{*}{ الأبعاد } \\
\hline & & & لا اتفق & 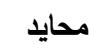 & أتفق & & \\
\hline 90.586 & 0.5331 & 2.7176 & 7.575 & 13.05 & 79.35 & RO1-RO4 & لجاهزية التنظيمية \\
\hline 90.958 & 0.4972 & 2.7287 & 7.360 & 12.36 & 80.24 & RH1-RH5 & جاهزيـة الموارد البشرية \\
\hline 77.680 & 0.7140 & 2.3304 & 22.36 & 22.26 & 55.4 & RT1-RT5 & لجاهزيـة التكنولوجية \\
\hline 83.940 & 0.6584 & 2.5182 & 13.95 & 20.22 & 65.8 & RL1-RL4 & لجاهزية القانونية \\
\hline 85.791 & 0.6007 & 2.5737 & 12.81 & 16.92 & 70.17 & & لمعدل العام \\
\hline
\end{tabular}

المصدر: من إعداد الباحثين بالاعتماد على مخرجات برنامج (SPSS V.23). ثانياً - وصف وتثخيص التنمية المستدامة: يثير الجدول (0) إلى أن المعدل العام لإجابات الأفراد المبحوثين

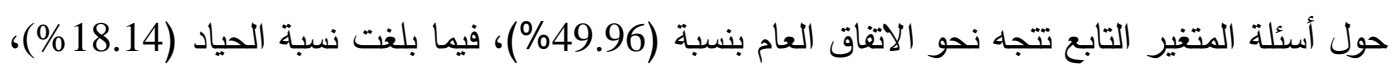
في حين كانت نسبة عدم الاتفاق (31.9\%)، وتعزيزاً لتلك النسب، بلغ الوسط الحسابي العام لهذا المتغير

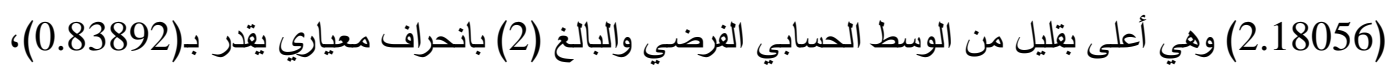

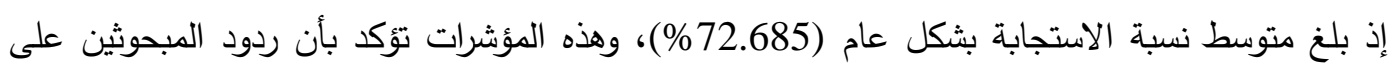

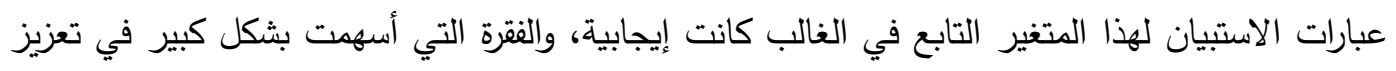
وإغناء المتغير التابع هذا هو (SD1) والمتمثلة بسعي ديوان محافظة نينوى إلى تحقيق التتمية المستدامة 


\section{انعكاسات أبعاد بحاهزية الحكومة الالكترونية.}

الاعربحي والسمان

بمجالاتها الثلاثة (الاجتماعية، والاقتصادية والبيئية)، إذ بلغت نسبة الاتفاق (79.4\%)، وكانت نسبة الاستجابة (90.420\%) وبوسط حسابي يقدر بـ(2.7126) وبانحراف معياري مقداره (0.60684) ، وهذا دليل على وجود انسجام في إجابات المبحوثين، إذ تثير إلى اتفاق المبحوثين بدرجة واضحة حول هذبان هذه الفقرة (SD1) وفقاً لوجهة نظرهم الثخصية.

جدول(0) المعدل العام والتوزيعات التكرارية والاوساط الحسابية والانحرافات المعيارية و نسبة الاستجابة للمتغير المعتمد (التنمية المستدامة)

\begin{tabular}{|c|c|c|c|c|c|c|c|c|c|}
\hline \multirow[t]{2}{*}{ نسبة الاستجابة } & \multirow{2}{*}{ الانحراف } & \multirow{2}{*}{ الحسابي } & \multicolumn{2}{|c|}{ لا اتفق } & \multicolumn{2}{|c|}{ محايد } & \multicolumn{2}{|c|}{ اتفق } & \multirow{2}{*}{ العبارات } \\
\hline & & & $\%$ & التكرار & $\%$ & التكرار & $\%$ & التكرار & \\
\hline 90.420 & 0.60684 & 2.7126 & 8.1 & 20 & 12.6 & 31 & 79.4 & 196 & SD1 \\
\hline 66.936 & 0.88800 & 2.0081 & 38.9 & 96 & 21.5 & 53 & 39.7 & 98 & SD2 \\
\hline 68.690 & 0.89735 & 2.0607 & 37.2 & 92 & 19.4 & 48 & 43.3 & 107 & SD3 \\
\hline 69.770 & 0.89000 & 2.0931 & 35.2 & 87 & 20.2 & 50 & 44.5 & 110 & SD4 \\
\hline 67.610 & 0.91243 & 2.0283 & 40.1 & 99 & 17.0 & 42 & 42.9 & 106 & SD5 \\
\hline 72.685 & 0.83892 & 2.1805 & 31.9 & & 18.14 & & 49.9 & & المعدل \\
\hline
\end{tabular}

المصدر: من إعداد الباحثين بالاعتماد على مخرجات برنامج (23. SPSS V).

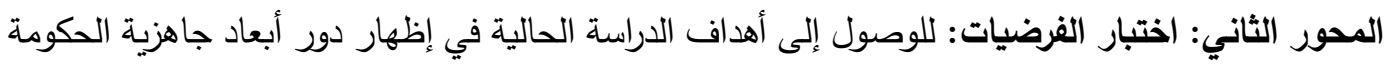

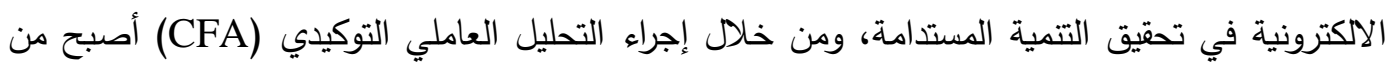

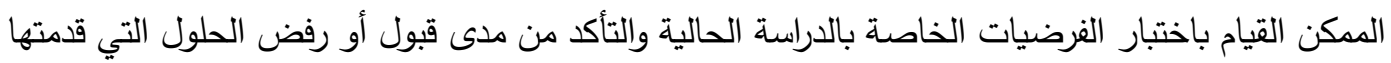
الدراسة للمشاكل التي طُرحَت والمحددة في المنهجية سابقاً وعلى النحو الآتي:

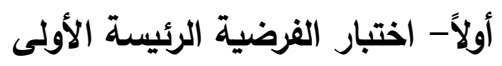

H0.1

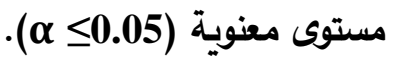


من أجل التحقق من صحة الفرضية تم صياغة أنموذج المعادلة البنائية الخاص بإثبات أو نفي صحة هذه الفرضية، إذ أظهرت نتائج التحليل الإحصائي الخاصة بنمذجة المعادلة البنائية معنوية الأنموذج المصدم لاختبار الفرضية الرئيسة الثانية، وكما موضحة في الثكل (Y) الخاص بندذجة المعادلات البنائية لمتغيرات الدراسة مجتمعة والتي تبين وجود علاقة ارتباط بين الحكومة الاكترونية بأبعادها الأربعة مجتمعة (الجاهزية لتنظيمية، جاهزية الموارد البشرية، الجاهزية التكنولوجية، الجاهزية القانونية) والتتمية المستدامة.

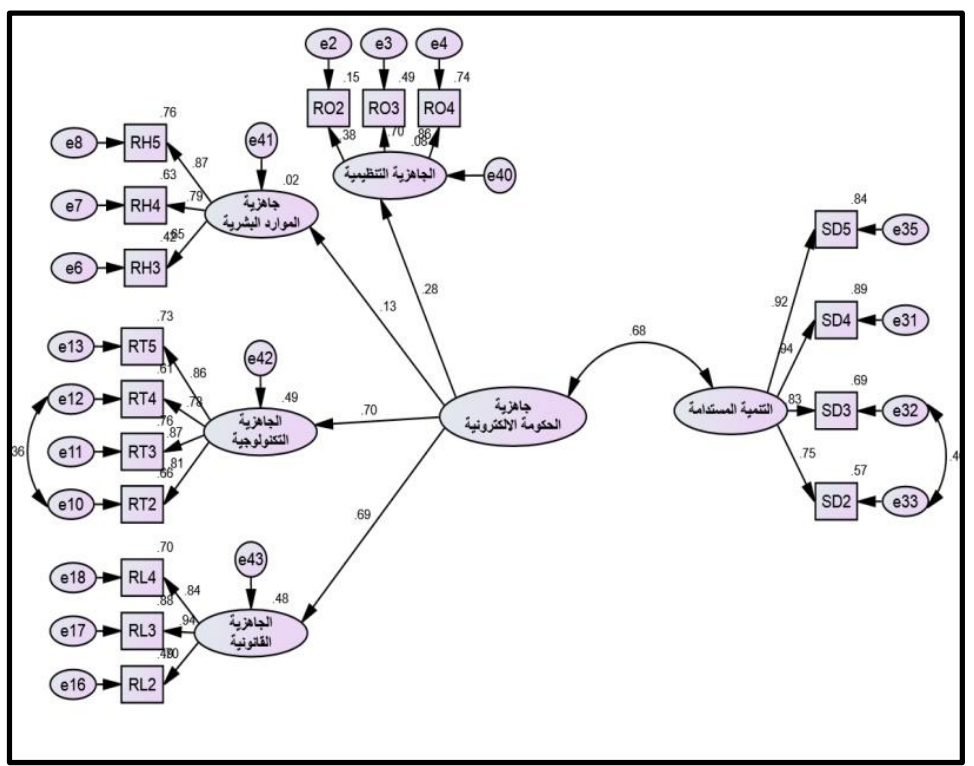

الشكل (ץ) الأنموذج البنائي لعلاقة الارتباط بين أبعاد جاهزية الحكومة الاكترونية مجتمعة والتنمية المستدامة المصدر: مخرجات البرمجية الإحصائية (Amos. v 24) وبعد الاطلاع على مؤشرات الجدول (†) يظهر أن هناك علاقة ارتباط إيجابية قوية بين المتغير الإنية المستقل (جاهزية الحكومة الالكترونية) والمتغير المعتمد (التتمية المستدامة)، حيث بلغت الترات القيمة التقديرية المتتبأ بها (0.684)، من هنا نرفض الفرضية الصفرية ونقبل الفرضية البديلة التي تتص على التى

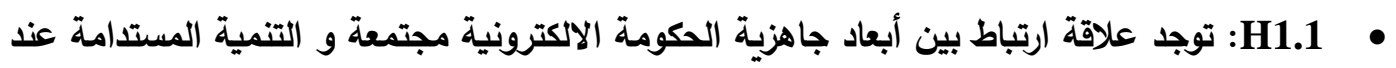

$$
\text { مستوى معنوية (1) }
$$

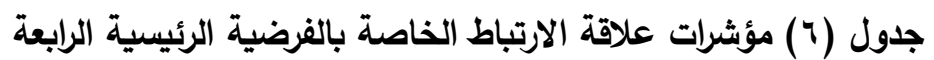

\begin{tabular}{|c|c|c|c|}
\hline التقدير & المتغير المؤثر به & مسار التأثير & لمتغير المؤثر \\
\hline 0.684 & التتمية المستدامة & 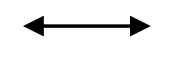 & 2 الحكومة الالكترونية \\
\hline
\end{tabular}

المصدر: من اعداد الباحثين بالاعتماد على مخرجات البرمجية الإحصائية (Amos. v 24) ثانياً - اختبار الفرضيات الفرعية للفرضية الرئيسة الثانية 


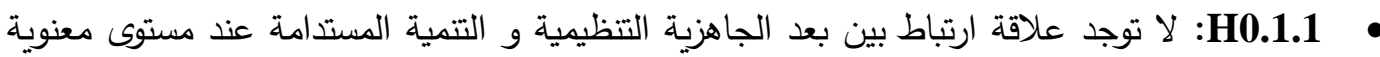

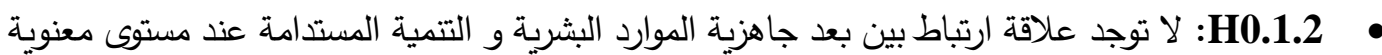

ه0.1.3 لا توجد علاقة ارتباط بين بعد الجاهزية التكنولوجية و التتمية المستدامة عند مستوى معنوية

• لا توجد علاقة ارتباط بين بعد الجاهزية القانونية والتتمية المستدامة عند مستوى معنوية

.$(\alpha \leq 0.05)$

ومن أجل التأكد من صحة هذه الفرضيات الفرعية والتحقق من مدى مطابقتها تم صياغة الأنموذج الخاص لإثبات أو نفي صحة هذه الفرضيات الفرعية، والثكل (ب) يوضح ذلك، وأظهرت نتائج التحليل

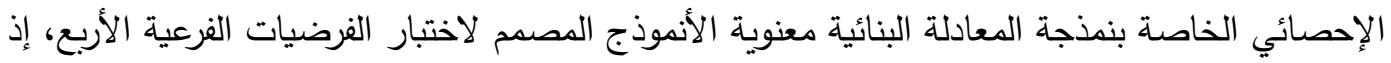

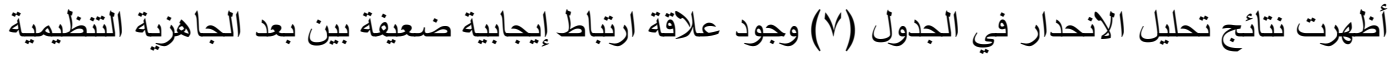

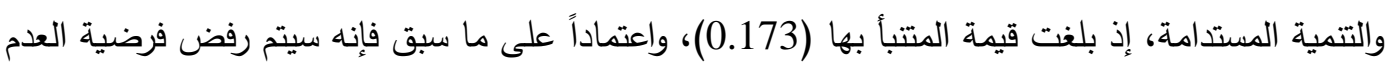

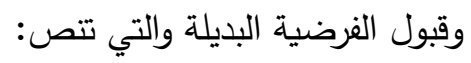

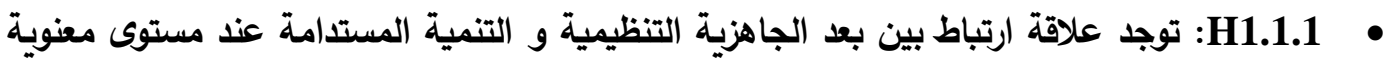
.$(\alpha \leq \mathbf{0 . 0 5})$

ويوضح الجدول (V) نتائج التحليل الإحصائي الخاصة بندذجة المعادلة البنائية إلى وجود علاقة

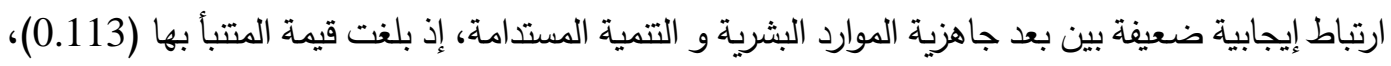

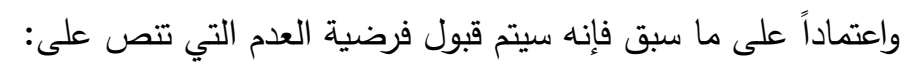

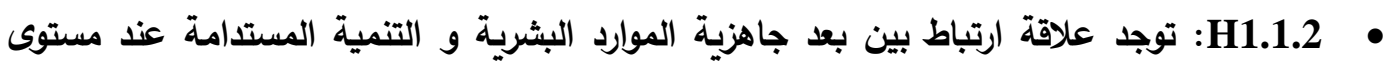

$$
\text { معنوية (م)0.05) }
$$

وأوضحت نتائج التحليل والموجودة في الجدول (V) بوجود علاقة ارتباط إيجابية متوسطة بين الجاهزية

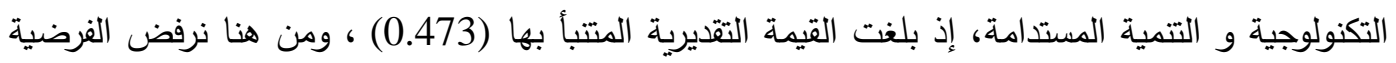
الصفرية ونقبل الفرضية البديلة والتي تتص: و النصاه

• • H1.1.3: توجد علاقة ارتباط بين بعد الجاهزية التكنولوجية و التنمية المستدامة عند مستوى معنوية .( $\alpha \leq \mathbf{0 . 0 5})$

كما نلاحظ في الجدول (V) بوجود علاقة ارتباط إيجابية متوسطة بين الجاهزية القانونية و التتمية

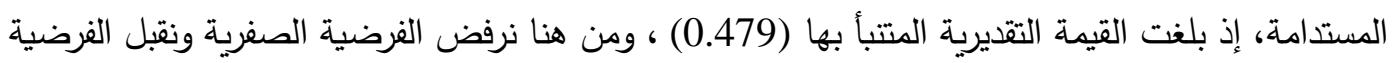

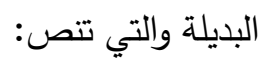


• H1.1.4: توجد علاقة ارتباط بين بعد الجاهزية القانونية و التنمية المستدامة عند مستوى معنوية .$(\alpha \leq 0.05)$

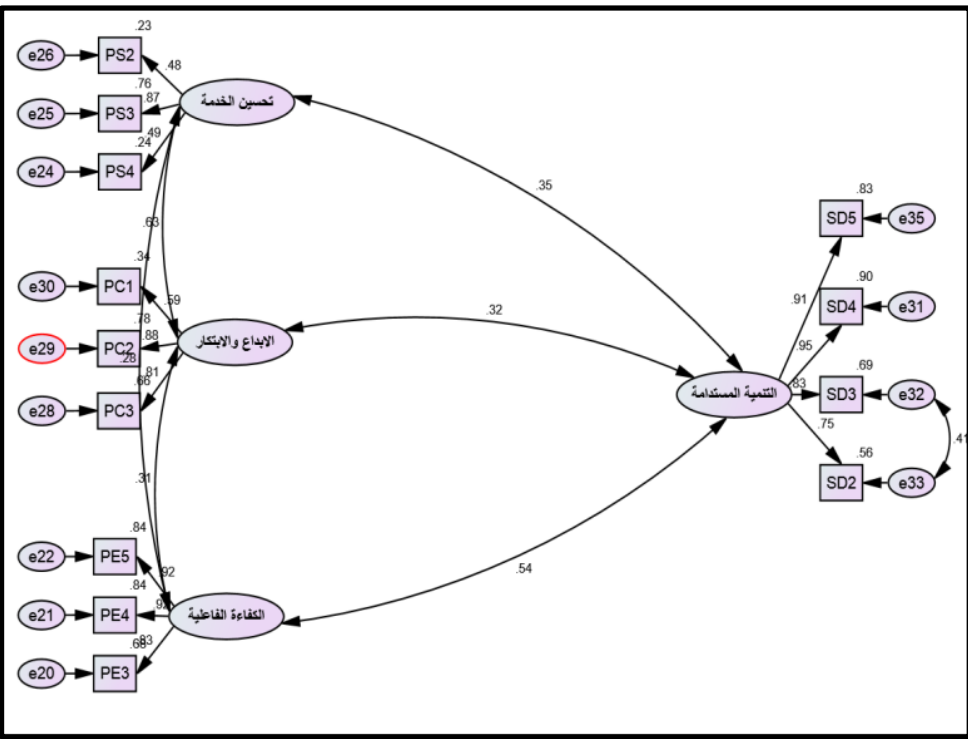

الثكل (ץ) الأنموذج البنائي لعلاقة الارتباط بين أبعاد جاهزية الحكومة الاككترونية منفردة و التنمية

المصدر: مخرجات البرمجية الإحصائية (Amos. v 24). جدول (V) مؤشرات علاقة الارتباط الخاصة بالفرضيات الفركاتهية

\begin{tabular}{|c|c|c|c|}
\hline التقدير & المتغير المؤثر به & مسار التأثير & المتغير المؤثر \\
\hline 0.173 & التتمية المستدامة & $\longleftrightarrow$ & الجاهزية التتظيمية \\
\hline 0.113 & التتمية المستدامة & $\longleftrightarrow$ & جاهزية الموارد البشرية \\
\hline 0.473 & التتمية المستدامة & $\longleftrightarrow$ & الجاهزية التكنولوجية \\
\hline 0.479 & التتمية المستدامة & $\longleftrightarrow$ & الجاهزية القانونية \\
\hline
\end{tabular}

المصدر: من إعداد الباحثين بالاعتماد على مخرجات البرمجية الإحصائية (Amos. v 24) ثالثاً- اختبار الفرضية الرئيسة الثانية

H0.2 لا يوجد أثر لأبعاد جاهزيـة الحكومة الاكترونية مجتمعة في التنمية المستدامة عند مستوى التهية

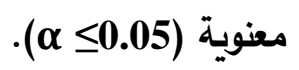

من أجل التحقق من صحة الفرضية تم صياغة أنموذج المعادلة البنائية الخاص بإثبات أو نفي صحة هذه الفرضية، إذ أظهرت نتائج التحليل الإحصائي الخاصة بنمذجة المعادلة البنائية معنوية الأنموذج المصدم

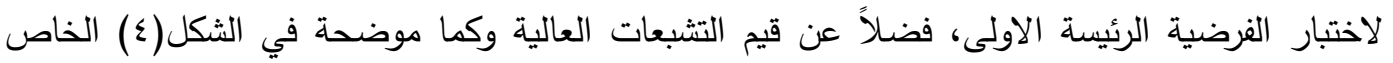
بنمذجة المعادلات البنائية لمتغيرات الدراسة مجتمعة والتي تبين وجود أثر لجاهزية الحكومة الاككترونية 
بأبعادها الأبعة مجتمعة (الجاهزية التظيمية، جاهزية الموارد البشرية، الجاهزية التكنولوجية، الجاهزية

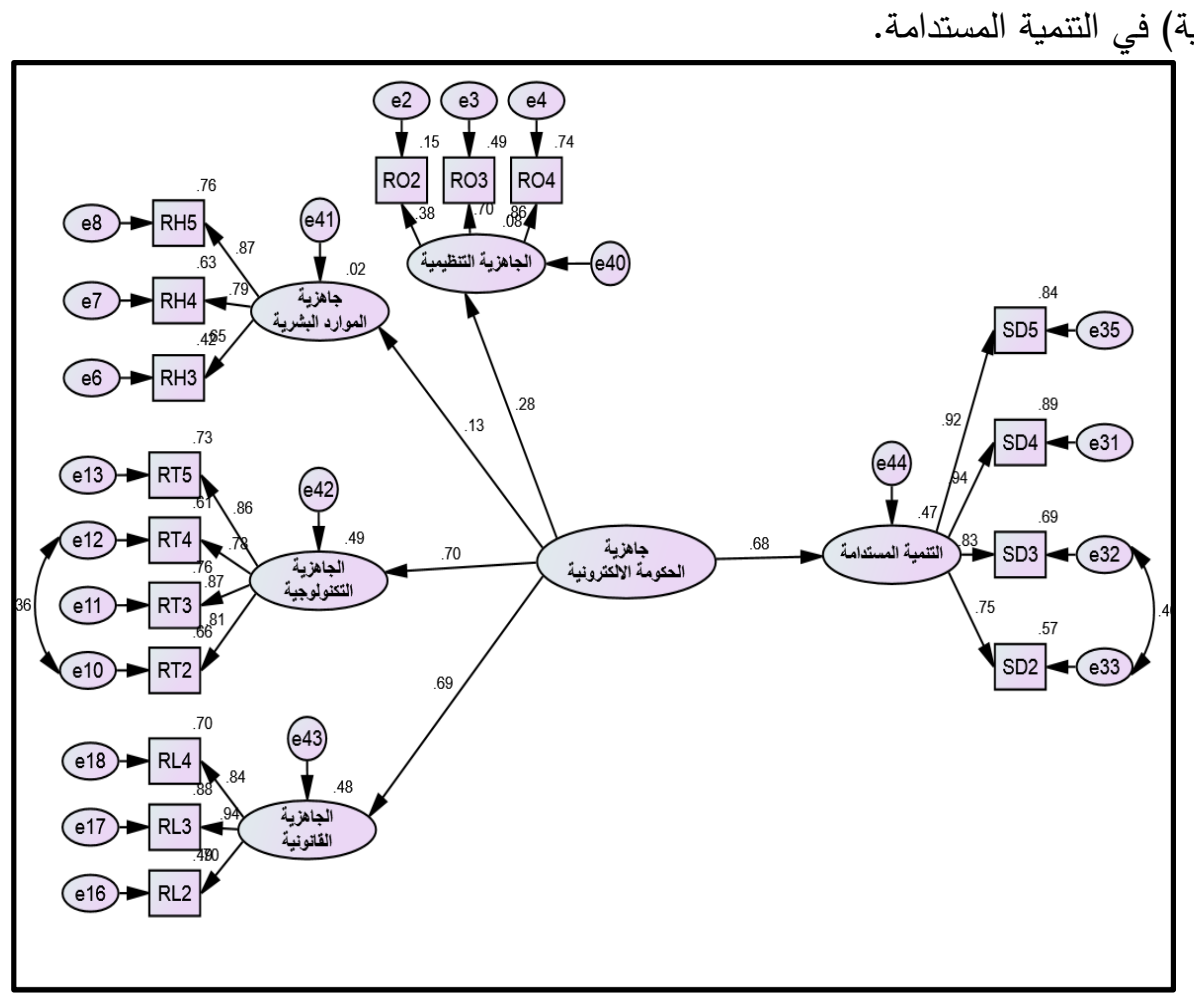

الثكل (ء ) الأنموذج البنائي لعلاقة الأثر لأبعاد جاهزية الحكومة الاكترونية مجتمعة في التنمية المستدامة المصدر : مخرجات البرمجية الإحصائية (Amos. v 24).

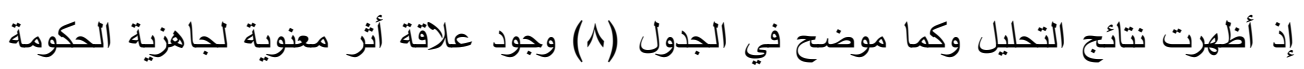

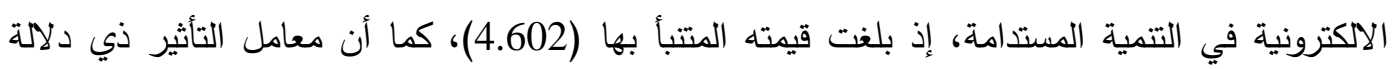
احصائية، إذ بلغت قيمة (C.R) مقدار (3.193) وهي قيمة مقبولة، بدلالة قيمة (P) والتي ظهرت (0.001) وهي قيمة أقل من القيمة المعنوية (0.05)، مما يدل على أنه كلما كانت الحكومة الالكترونية ذات جاهزية

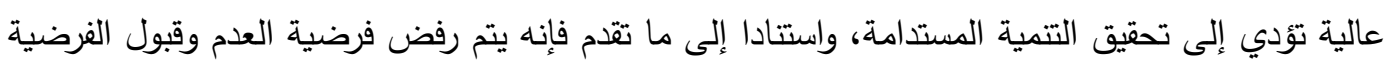
البديلة التي مفادها:

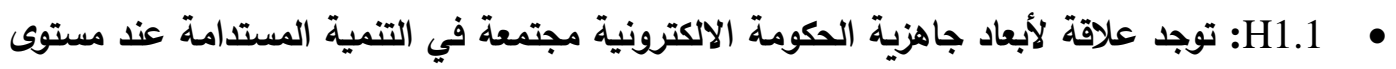

$$
\text { معنوية (م) }
$$

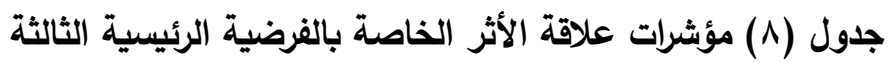

\begin{tabular}{|c|c|c|c|c|c|c|}
\hline $\mathrm{P}$ & C.R. & S.E. & التقدير & المتغير المؤثر به & مسار التأثير & المتغير المؤثر \\
\hline 0.001 & 3.193 & 1.441 & 4.602 & التتمية المستدامة & & جاهزية الحكومة الالكترونية \\
\hline
\end{tabular}

المصدر: من إعداد الباحثين بالاعتماد على مخرجات البرمجية الإحصائية (Amos. v 24) 


\section{انعكاسات أبعاد بحاهزية الحكومة الالكترونية. \\ الاعربحي والسمان}

ثانياً - اختبار الفرضيات الفرعية للفرضية الرئيسة الثانية

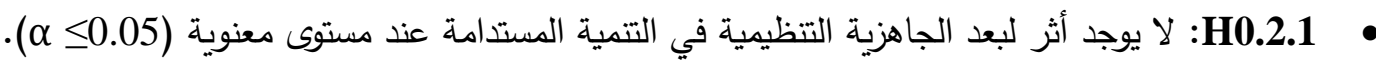

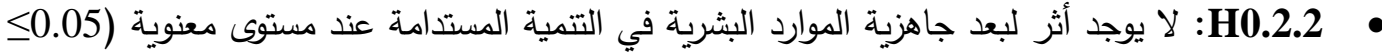

$\cdot(\alpha$

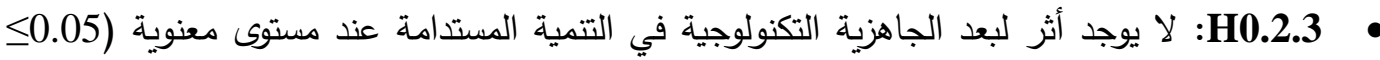

$\cdot(\alpha$

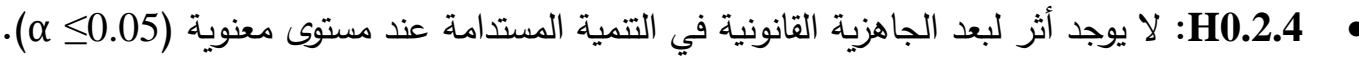
ومن أجل التأكد من صحة هذه الفرضيات الفرعية والتحقق من مدى مطابقتها تم صياغة الأنموذج

الخاص لإثبات أو نفي صحة هذه الفرضيات الفرعية، والثكل (0) يوضح ذلك، وأظهرت نتائج التحليل الإحصائي الخاصة بنمذجة المعادلة البنائية في الجدول (9) عدم وجود علاقة أثر معنوية لبعد الجاهزية التتظيمية في التتمية المستدامة، إذ بلغت قيمة المتتبأ بها (0.086)، بدلالة قيمة (P) التي بلغت (0.464) وهي قيمة أكبر من القيمة المعنوية (0.05)، ما يدل على وجود ضعف في الجاهزية التتظيمية للحكومة

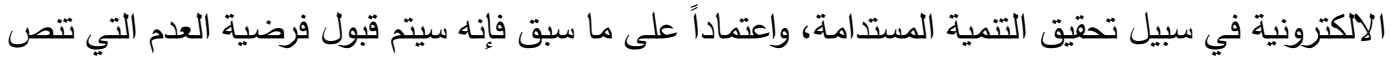

على:

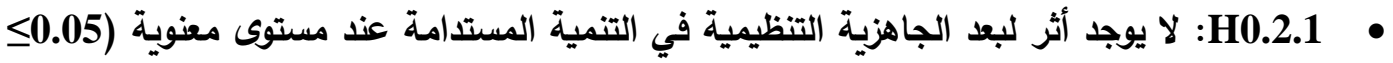

$\cdot(\alpha$

ويوضح الجدول (9) نتائج التحليل الإحصائي الخاصة بنمذجة المعادلة البنائية إلى عدم وجود علاقة

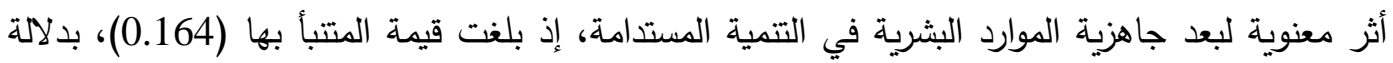

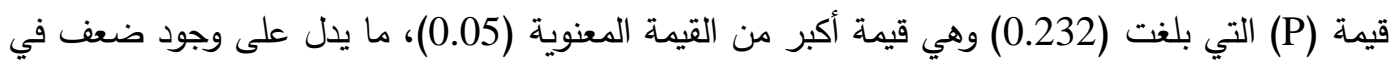
جاهزية الموارد البشرية للحكومة الالكترونية في سبيل تحقيق التتمية المستدامة، واعتماداً على ما سبق فإنهانه سيتم قبول فرضية العدم التي تنص على:

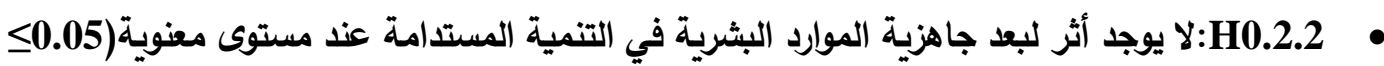

وأوضحت نتائج التحليل والموجودة في الجدول (9) وجود علاقة أثر معنوية للجاهزية التكنولوجية في

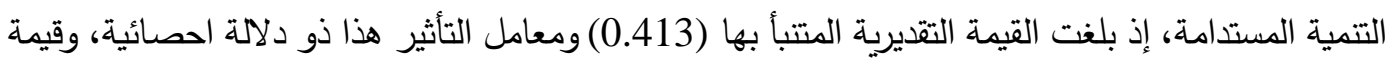
(C.R.) اهتمام في الجاهزية التكنولوجية لغرض تحقيق التتمية المستدامة، من هنا نرفض الفرضية الصناف الصنرية ونقبل الفرضية البديلة والتي تتص:

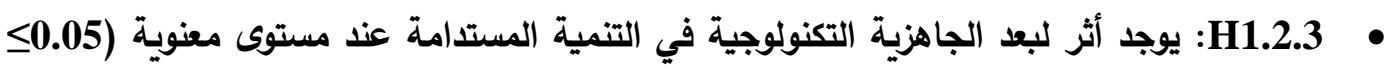




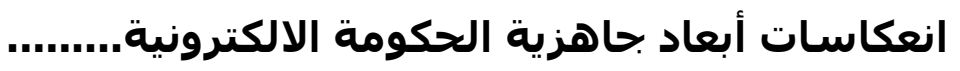 \\ الاعربحي والسمان}

كما نلاحظ في الجدول (9) بوجود علاقة أثر معنوية للجاهزية القانونية في التتمية المستدامة، إذ بلغت

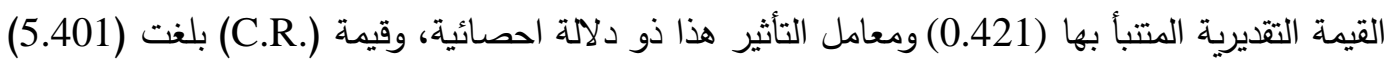
بدلالة قيمة (P) البالغة (0.000) وهي أقل من (0.05)، وهذا يدل على حتمية الجاهزية القانونية للحكومة الالكترونية في تحقيق التتمية المستدامة، ومن هنا نرفض الفرضية الصفرية ونقبل الفرضية البديلة والتي تتص:

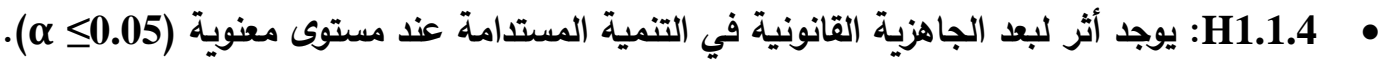
الثكل (0) الأنموذج البنائي لعلاقة الأثر لأبعاد جاهزية الحكومة الالكترونية منفردة في التنمية المستدامة

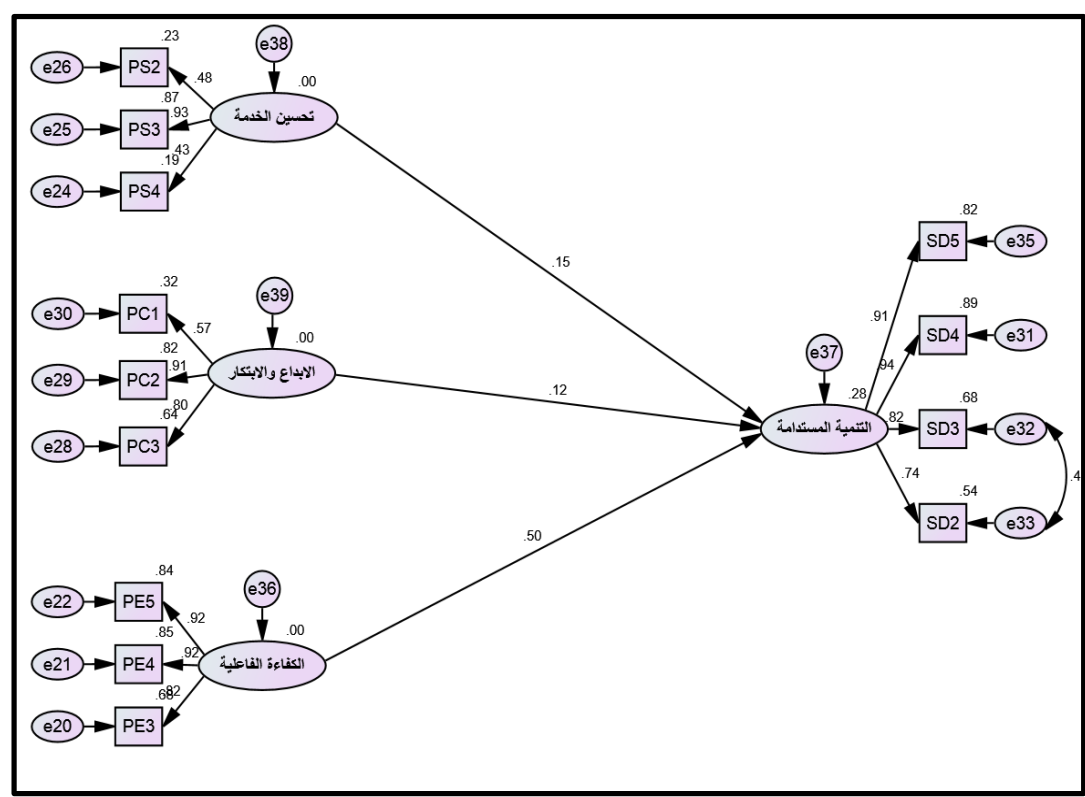

المصدر: مخرجات البرمجية الإحصائية (Amos. v 24). جدول (9 ) مؤشرات علاقة الأثر الخاصة بالفرضيات الفرعية الثانية

\begin{tabular}{|c|c|c|c|c|c|c|}
\hline $\mathbf{P}$ & C.R. & S.E. & التقدير & المتغير المؤثر به & مسار التأثير & المتغير المؤثر \\
\hline 0.464 & 0.733 & 0.117 & 0.086 & التتمية المستدامة & & الجاهزية التتظيمية \\
\hline 0.232 & 1.195 & 0.137 & 0.164 & التتمية المستدامة & & جاهزية الموارد البشرية \\
\hline$* * *$ & 5.287 & 0.078 & 0.413 & التتمية المستدامة & & الجاهزية التكنولوجية \\
\hline$* * *$ & 5.401 & 0.078 & 0.421 & التتمية المستدامة & & الجاهزية القانونية \\
\hline
\end{tabular}

المصدر: من اعداد الباحثين بالاعتماد على مخرجات البرمجية الإحصائية (Amos. v 24)

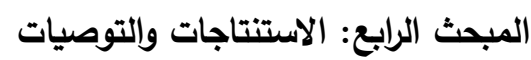




\section{انعكاسات أبعاد بحاهزية الحكومة الالكترونية......... \\ الاعرجي والسمان}

المحور الأول - الاستنتاجات: تمثل الاستتتاجات المطروحة الحصيلة الفكرية والتحليلية للدراسة الحالية، والتي

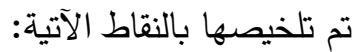

1. أظهرت نتائج وصف وتثخيص متغيرات الدراسة أهمية الأبعاد الأربعة لجاهزية الحكومة الالكترونية في

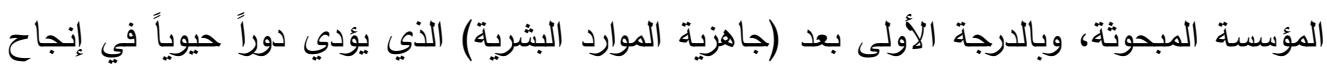
تطبيق مشروع الحكومة الالكترونية.

r. أظهرت نتائج وصف وتثخيص متغيرات الدراسة بوجود سعي من قبل المؤسسة المبحوثة في تحقيق التتمية المستدامة، إلا أنها تفتتر إلى مؤشرات واضحة لتحقيق التتمية المستدامة، وهذا ما أثبتته الفقرة رقم

$$
\text { (SD2) }
$$

r. إسهام أبعاد جاهزية الحكومة الالكترونية على المستوى الكلي في التأثير وبشكل مباشر وإيجابي في تحقيق التتمية المستدامة على صعيد محافظة نينوى وبشكل عام، وهذا يفسر امتلاك ديوان محافظة نينوى فئي الإمكانات والقدرات الالكترونية التي تسهم في تحقيق التتمية المستدامة.

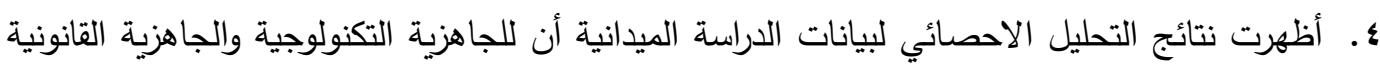

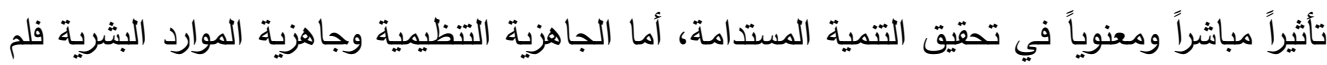
يظهر لهما تأثير في تحقيق التتمية المستدامة، وهذا يعني قدرة المؤسسة المبحوثة (ديوان محافظة نينوى)

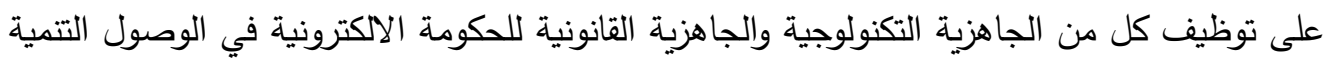
المستدامة وتحقيق أهدافها، إلا أنها تعاني من صعوبات في العمليات التتظيمية والإدارية وإدارة الموارد

$$
\text { البشرية وتوعية الجمهور لتحقيق التنمية المستدامة. }
$$

ه. أظهرت نتائج البحث الميداني، وجود ضعف في إدرالك عينة الدراسة للتمية المستدامة وجوانبها الثلاثة، وعدم وجود آلية أو فريق يعنى في المجال المهم و الحيوي، على الرغم من وجود مشروعات واستراتيجيات ينفذها ديوان محافظة نينوى، ويعزى هذا الامر لما مرت به محافظة نينوى في السنوات السابقة، إذ تم

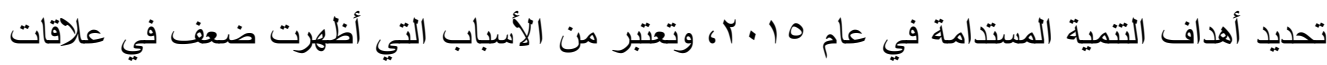

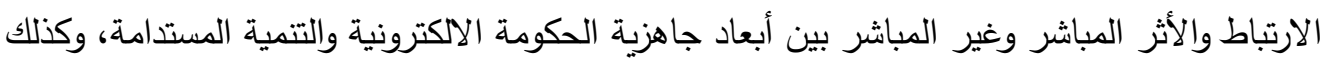

$$
\text { الأداء المؤسسي والتتمية المستدامة. }
$$

المحور الثاني- التوصيات : بناء على الاستتتاجات التي توصلت إليها الدراسة نقترح على المؤسسة المبحوثة

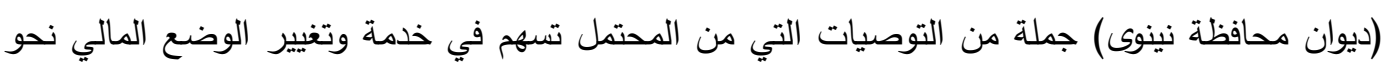

$$
\text { الأفضل وتتمثل في الآتي: }
$$

1. تقديم الدعم الثامل والكامل وبخطى فعلية من أجل انجاز وتهيئة أبعاد الحكومة الاككترونية بشكل متوازٍ

وتوظيف آثارها التتموية للجوانب (الاجتماعية والاقتصادية والبيئية) والمتمثلة بالتنمية المستدامة.

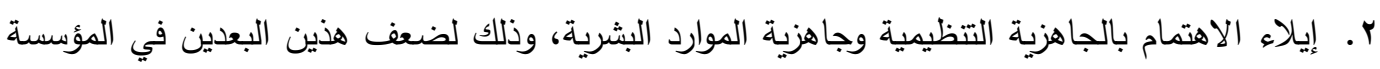

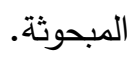




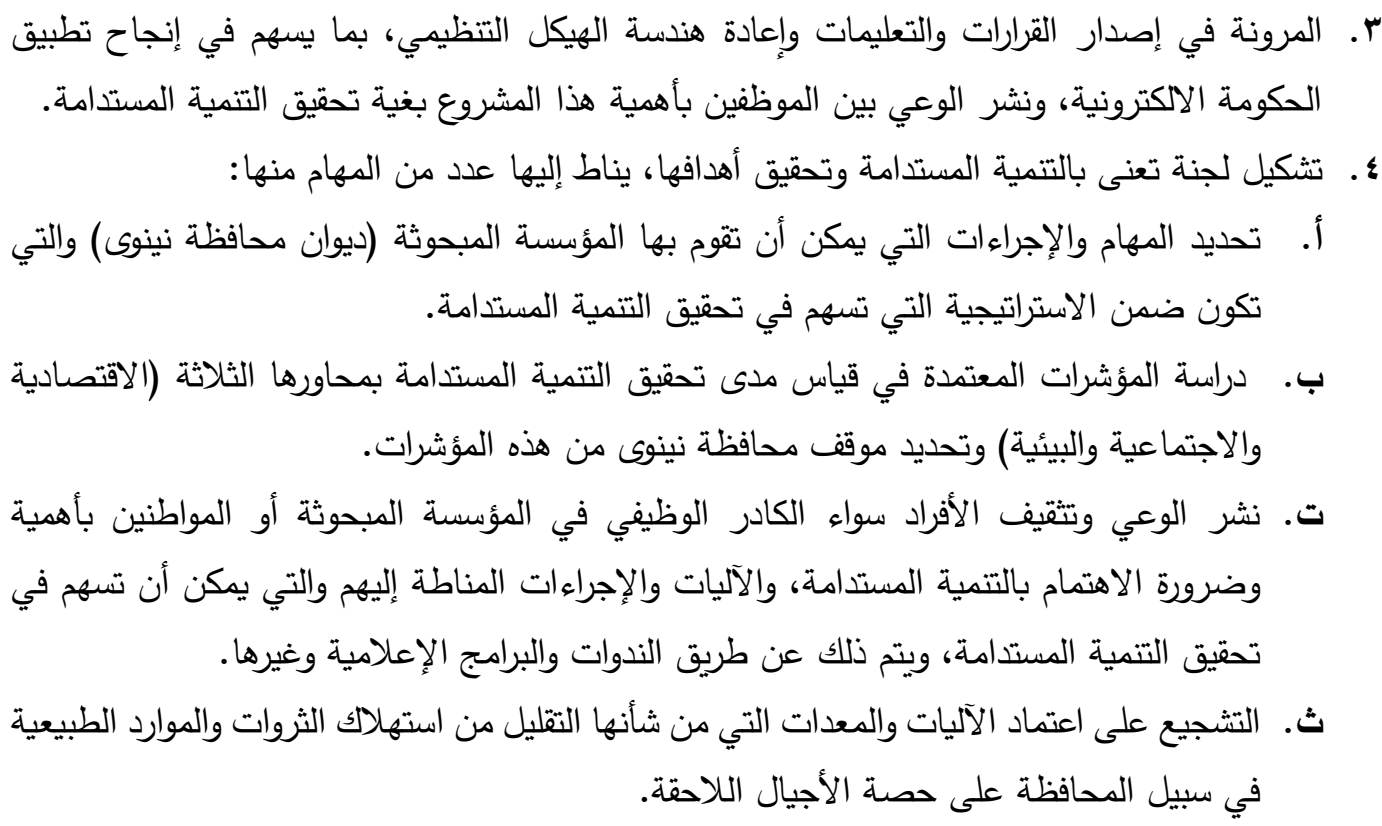

\section{References:}

ALbla, Abdullah, H., (2018), " Role of the E_ Readiness for The Adoption Of E_ Banking Application "Survey Study at Mosul Bank For Development and Investment", Iraqi Journal of Information Technology, Volume 8, Issue 13.

Al-Samman، Thaeir,A., (2008), "The Integration between Agile Manufacturing Strategies and Lean Manufacturing Techniques and their Impact on Fostering Operational Performance An Application Study in Selected Industrial Companies in the City of Mosul, Ph. D. thesis, The College of Administration and Economics, University of Mosul.

Hanan, Brahmi, (2015), "The crime of falsifying the official administrative document of an informational nature, $\mathrm{PhD}$ thesis of sciences specializing in criminal law, Faculty of Law and Political Science, University of Mohamed Khaider, Biskra, Algeria.

Hashim, Abdullah, and Abdulhaq, Abdullah, (2019), "IT capabilities in achieving sustainable development of the education sector in Iraq, An Exploratory Study of Several Colleges of the University of Mosul", Baghdad College of Economic Sciences Journal, Issue of the Eighth International Scientific Conference (2019).

Bou Marwan, Sumaya, (2014), "E-government and its role in improving the performance of government departments, a comparative study", first edition, Law and Economics Library, Riyadh, Saudi Arabia.

Al-Enzee, Eman, M., (2013),"Possibility of implementing the electronic government to Reinforcement decision making process A suggested system in directorate of sociality care/ Nineveh", Master Thesis, Management Information Systems, College of Administration and Economics, University of Mosul. 


\section{انعكاسات أبعاد بحاهزية الحكومة الالكترونية....................... \\ الاعربحي والسمان}

Al-Rizo, Hassan Muzhaffar, (2012), "The electronic readiness of Arab countries and their potential implications for opportunities to activate the knowledge economy environment," first edition, Center for Arab Unity Studies, Beirut, Lebanon

Al-Habal, Ghalia, (2003), Sustainable Development, a study prepared for a higher diploma, Faculty of Environmental Engineering, Postgraduate Studies, Damascus University, Damascus, Syria.

Abdul Salam, Muhammad Al-Sayed (1998), "Food Security for the Arab World", a monthly cultural book series issued by the National Council for Culture, Arts and Literature, The World of Knowledge, Kuwait.

Abdullah, Abdel Khaleq (1998), "Sustainable development and the relationship between environment and development", Center for Arab Unity Studies, Arab Future Book Series, First Edition, Beirut, Lebanon.

Haffaf, Souad and Boutiaf, Malika, (2015), The Social Dimension of Sustainable Development in Algeria in Light of Low Oil Prices, Missiba Ben Bou Ali Chlef University - Alger

Abu El-Nasr, Medhat and Mohamed, Yasmine Medhat, (2017), Sustainable Development: Its Concept - Dimensions - Its Indicators, The Arab Group for Training and Publishing, First Edition, Deposit Number: 1509/2017, 8 A, Ahmed Fakhry Street, Nasr City, Cairo.

Abu El-Maati, Maher, (2014), Modern Trends in Social Welfare and Social Work, Modern University Office, First Edition, Egypt.

E-GOVERNMENTSURVEY 2018 GEARING E-GOVERNMENT TO SUPPORT TRANSFORMATION TOWARDS SUSTAINABLE AND RESILIENT SOCIETIES" (2018), Department of Economic and Social Affairs, United Nations, New York.

Steve, Cedric, Bizimana, 2020, "E-government Readiness Assessment for Government institutions in Burundi", International Journal of European Studies. Vol. 4, No. 1, pp. 1-8. doi: 10.11648/j.ijes.20200401.11.

Jeffrey D., et al.(2019), Handbook of Green Finance Energy Security and Sustainable, company Springer Nature Singapore Pte Ltd.

Maiye, Ariyo and McGrath, Kathy, (2010) "ICTs AND SUSTAINABLE DEVELOPMENT: A CAPABILITY PERSPECTIVE", Americas Conference on Information Systems (AMCIS) $2010 \quad$ Proceedings. 541. http://aisel.aisnet.org/amcis2010/541

Shareef, M., Ojo, A., and Janowski, T.,(2008), "A Readiness Assessment Framework for e-Government Planning - Design and Application", National Centre for Information Technology, Republic of Maldives.

Al-Omari, A. and Al-Omari, H.,(2006), " E-Government Readiness Assessment Model" ,Journal of Computer Science 2 (11): 841-845, Amman, Jordan.

Alshaher, A., (2013), THE MCKINSEY 7S MODEL FRAMEWORK FOR ELEARNING SYSTEM READINESS ASSESSMENT, International Journal of Advances in Engineering \& Technology, Vol. 6, Issue 5, pp. 1948-1966.

TANMIYAT AL-RAFIDAIN (P-ISSN: 1609-591X; E-ISSN: 2664-276X) تنمية الرافدين 


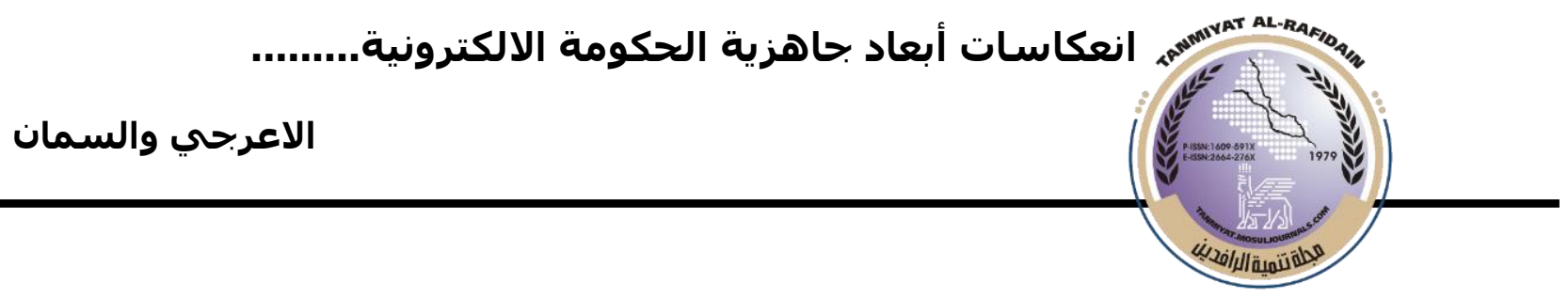

UNESCO,(2012), Education for Sustainable Development in Action UNESCO Education Sector, Source book, the United Nations Educational, Scientific and Cultural Organization 7, place de Fontenoy, 75352 Paris 07 SP, France.

Reed, D.(2003),EXECUTIVE SUMMARY of Structural Adjustment, the Environment and Sustainable Development, Macroeconomics for Sustainable Development Program Office (MPO),WWF International, 1250 24th Street, N.W. Washington, D.C. 20037-1175,Etats-Unis d'Amérique.

Maria,L., Vasiliki, L., Aikaterini, O.(2015), Sustainable Development and the Environment, Introduction to Sustainable Development, A brief handbook for students by students, International Hellenic University.

Hagen, B., Nassar, C., \& Pijawka, D. (2017). The Social Dimension of Sustainable Neighborhood Design: Comparing Two Neighborhoods in Freiburg, Germany. Urban Planning, 2(4), 64-80. 